\title{
Neural synchrony in cortical networks: history, concept and current status
}

\author{
Peter J. Uhlhaas 1,2, Gordon Pipa, ${ }^{1,3}$, Bruss Lima' , Lucia Melloni' ${ }^{1}$, Sergio Neuenschwander ${ }^{1}$, Danko Nikolic ${ }^{1,3}$ \\ and Wolf Singer ${ }^{1,3 *}$
}

\author{
Department of Neurophysiology, Max Planck Institute for Brain Research, Frankfurt am Main, Germany \\ 2 Laboratory for Neurophysiology and Neuroimaging, Department of Psychiatry, Johann Wolfgang Goethe Universität, Frankfurt am Main, Germany \\ ${ }^{3}$ Frankfurt Institute for Advanced Studies, Johann Wolfgang Goethe Universität, Frankfurt am Main, Germany
}

\section{Edited by:}

Rui M. Costa, Champalimaud

Neuroscience Programme, Instituto

Gulbenkian de Ciência, Portugal

Reviewed by:

Miles A. Whittington, Newcastle

University, UK

Shih-Chieh Lin, Duke University

Medical Center, USA

${ }^{*}$ Correspondence:

Wolf Singer, Department of

Neurophysiology, Max Planck Institute for Brain Research, Deutschordenstr.

46, Frankfurt am Main 60528,

Germany.

e-mail: singer@mpih-frankfurt.mpg.de
Following the discovery of context-dependent synchronization of oscillatory neuronal responses in the visual system, the role of neural synchrony in cortical networks has been expanded to provide a general mechanism for the coordination of distributed neural activity patterns. In the current paper, we present an update of the status of this hypothesis through summarizing recent results from our laboratory that suggest important new insights regarding the mechanisms, function and relevance of this phenomenon. In the first part, we present recent results derived from animal experiments and mathematical simulations that provide novel explanations and mechanisms for zero and nero-zero phase lag synchronization. In the second part, we shall discuss the role of neural synchrony for expectancy during perceptual organization and its role in conscious experience. This will be followed by evidence that indicates that in addition to supporting conscious cognition, neural synchrony is abnormal in major brain disorders, such as schizophrenia and autism spectrum disorders. We conclude this paper with suggestions for further research as well as with critical issues that need to be addressed in future studies.

Keywords: synchrony, oscillations, gamma, cortex, cognition

\section{INTRODUCTION}

\section{NEURAL SYNCHRONY IN CORTICAL NETWORKS}

The cerebral cortex is a highly distributed system in which numerous areas operate in parallel, exchange their results via reciprocal reentrant connections and through self-organizing dynamics create coherent states that are equivalent with representations of sensory objects, decisions and programs for motor acts. The topology of the connectivity shares properties with small world networks which implies that there are certain areas which sustain more connections than others (hubs) but that there is no singular center where all information converges and which would be in a position to serve as a supraordinate coordinating center. This raises a number of important questions: i) how are the numerous computations occurring simultaneously in spatially segregated processing areas coordinated and bound together to give rise to coherent percepts and actions, ii) how are input signals selected for further processing and how are the results of sensory processes routed selectively across the densely connected network to executive structures and finally, iii) how are the often graded relations between simultaneously represented contents encoded. In principle, there are two different solutions to these problems. First, devoted architectures of connections that assume coordinating and binding functions through convergence and divergence of labelled lines, and second, dynamics that allow for the self-organization of ever changing spatio-temporal activity patterns on the backbone of fixed anatomical connections. These two strategies are not mutually exclusive but can coexist and complement one another. In the review we shall concentrate on the second strategy and here, in particular, on coordinating mechanisms that rely on the temporal patterning of neuronal activity and comprise phenomena such as self-generated network oscillations, synchronization and phase locking.

An important link between temporal patterning of neuronal activity and cortical computations was the discovery that oscillatory rhythms in the beta/gamma range $(20-80 \mathrm{~Hz})$ serve to establish precise and context dependent temporal relations - in this case synchrony - between distributed neural discharges. Gray et al. (1989) showed that action potentials generated by cortical cells align with the oscillatory rhythm which has the consequence that neurons phase locked to synchronized oscillations, synchronize their discharges with high precision. More recent evidence indicates that these oscillations are not only instrumental for the synchronization of neuronal discharges but can support also other consistent temporal relations by establishing systematic phase lags between the discharges of distributed neurons. In-vitro studies (Volgushev et al., 1998) and multi-site recordings in the visual cortex of cats (Fries et al., 2001b) provided evidence, that the oscillatory patterning of neuronal activity is an efficient mechanism to adjust the precise timing of spikes and is potentially a versatile mechanism to convert rate coded input to cells into a temporal code defined by the time of occurrence of spikes relative to the oscillation cycle (see also Fries et al., 2007).

Following the initial descriptions of context dependent synchronization in the visual cortex, numerous studies have been initiated in order to investigate the functional role of this phenomenon. These have demonstrated that response synchronization is a ubiquitous phenomenon in cortical networks and likely to serve a variety of different functions in addition to feature binding at early levels of sensory processing. 
Studies in the motion sensitive area MT of the visual cortex of awake monkeys (Kreiter and Singer, 1996), the optic tectum of pigeons (Neuenschwander et al., 1996), other cortical areas in the cat (Engel et al., 1991) and the retina (Neuenschwander and Singer, 1996) provided evidence that the oscillatory patterning of neuronal responses and the synchronization of these oscillations is highly sensitive to context. Combining multi-site recordings with the sectioning of the corpus callosum (Engel et al., 1991) and with developmental studies (König et al., 1993; Löwel and Singer, 1992) indicated that the long distance synchronization of oscillatory responses is mediated to a substantial extent by the network of reciprocal cortico-cortical connections. Multi-site recordings also provided evidence that synchronization occurs between widely distributed structures, such as the primary visual cortex, the optic tectum and the suprasylvian cortex (Brecht et al., 1998) and that it plays a role in the coordination of widely distributed functions as is required e.g. in sensory-motor processing (Roelfsema et al., 1997). This latter study indicated further that synchronized oscillatory activity is not only stimulus driven but also generated in anticipation of a visual discrimination task requiring fast motor responses. This observation led to the hypothesis that self-generated oscillatory activity in the beta- and gamma frequency range could be a correlate of focused attention and serve both modality specific selection of stimuli and the coordination of sensory and executive subsystems required for the execution of the anticipated task. A close relation between synchronization and input selection has also been found in experiments on binocular rivalry (Fries et al., 2001a).

The notion of an involvement of synchronized beta- and gamma oscillations in attention dependent processes agrees also with the evidence that there is a close relation between arousal, activated cortical states and the occurrence of high frequency oscillations. Gamma oscillations occur only with activated cortical states and require for their expression activation of muscarinic receptors in the cortex (Herculano-Houzel et al., 1999; Munk et al., 1996).

Taken together, the results suggested that synchronization enhances the saliency of the synchronized responses which can in turn be used for a variety of different operations. Joint increases of saliency favour joint selection and processing of signals which can in principle support attention dependent stimulus selection and feature binding.

The notion that the saliency of responses can be enhanced in a complementary way either by increases of discharge rate or by synchronization has later received direct support from experiments on apparent brightness perception (Biederlack et al., 2006).

\section{THE GENERATION OF NEURAL SYNCHRONY}

The discovery of synchronized oscillations has motivated a large number of in-vitro studies searching for the mechanisms that would generate these oscillations and this led to a re-evaluation of the functional role of inhibitory interneurons. Classically, inhibition has been considered as a mechanism for gain control, contrast enhancement and improvement of signal to noise ratios. In-vitro investigations of oscillating networks demonstrated, however, that the network of inhibitory interneurons plays a crucial role in the rhythmic pacing of neuronal activity. Through this additional function they assume a pivotal role in the temporal structuring and coordination of neuronal responses (Cardin et al., 2009; Sohal et al., 2009; for a review see Bartos et al., 2007).

These experimental results on oscillatory neuronal networks have motivated a large number of theoretical studies investigating the functional properties of networks capable of engaging in oscillations and stimulus dependent synchronization patterns. These studies provided deep insights into both the mechanisms that sustain oscillations and their synchronization as well as the putative functions of the temporal coding strategies that can be implemented in networks of coupled oscillators with essentially non-linear dynamics (Traub et al., 2004).

\section{STUDIES IN HUMAN SUBJECTS}

Non invasive electrophysiological methods such as EEG and MEG recordings register preferentially if not exclusively synchronized neuronal activity because they average over large populations of neurons. Thus, non-synchronized sources of activity tend to cancel out and synchronized signals are enhanced. This, together with the ease to perform demanding psychophysical experiments, is one of the reasons why investigations on synchronized oscillations and their putative function have been particularly rewarding in human subjects. These studies provided rapidly growing evidence for a close relation between synchronous oscillatory activity in the betaand gamma frequency range and a variety of cognitive functions such as perceptual grouping, focused attention, maintenance of contents in short term memory, poly-sensory integration, formation of associative memories and sensory motor coordination (for review see Singer, 2004; Tallon-Baudry, 2009).

\section{THE SCOPE OF THE PRESENT REVIEW}

Despite the abundant evidence for close correlations between oscillatory activity and a wide range of physiological and psychological phenomena, we are still far from understanding the full implications of the complex dynamics expressed in networks capable of engaging in oscillatory activity in various frequency bands. Numerous observations support the hypothesis that oscillations and synchrony are the backbone of temporal coding strategies but most of the evidence is still correlative in nature. Therefore, search for the functional implications of self-organized temporal patterning of neuronal activity is pursued by an increasing number of laboratories both at the experimental and theoretical level.

In the present review, we provide an update of results obtained in our lab that suggest novel mechanisms for the temporal coordination of distributed neuronal-responses and extend the scope of functions subserved by synchronized oscillatory activity. In this context we present results derived from animal experiments and mathematical simulations that provide explanations for the conundrum that nerve nets with finite conduction delays can synchronize with zero-phase lag (see 'Theoretical Approach' by Pipa). In addition, we discuss evidence that suggests that small delays between spikes occurring in synchrony with gamma oscillations contain stimulus related information (see 'Novel Empirical Results on Temporal Coding' by Nikolić). Next we present novel data on the relationship between attention, expectancy and gamma oscillations (see 'Building Expectations: New Vistas for Gamma Oscillations' by Lima and Neuenschwander) and the relation between phase locking of high frequency oscillations and consciousness (see 'Neural 
Synchrony as a Mechanism for Conscious Perception?' by Melloni). Finally, we investigate the relevance of synchronous oscillations in the context of psychiatric syndromes and report recent data which indicate that neural synchrony is abnormal in major psychiatric diseases such as schizophrenia and autism spectrum disorders that are associated with altered states of consciousness and dysfunctional cognition (see 'Neural Synchrony in Schizophrenia and Autism Spectrum Disorders' by Uhlhaas).

In the final part of the paper, we provide a perspective on open questions and challenges regarding the role of oscillations and synchrony and propose some empirical and theoretical strategies to cope with these questions.

\section{MECHANISM AND CODING PROPERTIES OF SYNCHRONIZATION}

One of the central problems regarding neural synchrony is the questions of how neurons can synchronise their responses with zero or near-zero phase lag synchrony. Early studies showed that zerophase lag synchronization can occur even between distant neuronal assemblies, raising the question of the mechanistic implementation of such phenomena. This is particularly relevant as the conduction delays in cortex make the occurrence of zero-phase lag synchrony difficult to accomplish.

In the following two sections, we will summarise recent work that has used computer simulations and mathematical approaches as well as multi-unit recordings to reveal novel mechanisms of zero lag and near-zero phase synchronization. We begin this section with what is known today about the low-level mechanisms responsible for generation of neuronal synchrony and for coding information by synchronized groups of neurons. The first section 'Theoretical Approach' is mostly an overview of the theoretical work, which revealed number of counterintuitive phenomena that could not be detected without the efforts based on mathematical analyses and computer simulations. The second section 'Novel Empirical Results on Temporal Coding' describes empirical work that helped elucidate the fundamentals of the synchrony-related operations within the neocortex.

\section{THEORETICAL APPROACH}

\section{Mechanisms for zero lag synchrony}

A special and challenging case of synchronization is zero time lag or zero phase synchronization in which the activity is modulated synchronously and either without any temporal delay or delay much smaller than the conduction delays ('near zero time lag' or 'near zero phase'). Remarkably, most neuronal synchrony was found to occur with such small phase lags between periodic or quasi periodic dynamics (Roelfsema et al., 1997). For simplicity, the first part focuses on zero time lag synchronization exclusively. Near time zero lag sychronization is covered in later sections. However, so far only very few and highly specialized models have been able to account for zero lag synchrony in networks coupled with delay. Especially in neuronal networks these delays can be substantial due to conduction times, synaptic delays and electrotonic propagation.

Maybe the simplest mechanism to induce zero phase synchronization is entrainment via common drive from a single source, i.e. from other cortical and sub-cortical areas (Steriade et al., 1993), that synchronizes the respective target cells. In local networks, pacemaker cells with intrinsic oscillatory activity can entrain the network activity in certain frequency range, i.e. chattering cells that have been discussed to be involved in generating stimulus driven gamma oscillations (Gray and McCormick, 1996). It is important to note that entrainment determines both the rhythm and the synchronization among the target cells. To study the limits of this simple mechanism in complex networks we modelled common drive and entrainment by an unidirectional coupling with an auxiliary hub (Huang and Pipa, 2007). The remarkable and generalizable finding was that entrainment via an auxiliary hub can always synchronize activity, and this occurs for any arbitrary coupling, e.g. linear or non-linear, for any network topology, and for a wide range of intrinsic properties of the neurons of the network. In addition, a lower bound was identified for the minimal coupling strength necessary for synchronization by entrainment. This minimal coupling strength was a function of the type of coupling between the hub and the network, but also of the properties of the network itself. Thus, the occurrence of synchronization can be controlled either by modifying the coupling of the auxiliary hub and therefore the strength of the entrainment and/or by modifications of the intrinsic properties of the neurons in the synchronized network or the coupling within this network. This suggests that even for a simple mechanism such as entrainment, the synchronization can be very dynamic, depending both on the strength of the entrainment and on numerous parameters of the complete system.

Another class of mechanisms for zero phase synchrony is emerging synchronization by network effects. This mechanism is very different from entrainment, since emerging properties are arising by the interaction of the elements in the network and cannot be explained by a single element or a subset of isolated elements alone. For zero phase synchronization based on such network effects several mechanisms have been proposed. Among these are recurrent inhibition, mutual excitation, mutual inhibition, gap junction coupling and synaptic spike doublet based coupling (Kopell et al., 2000; Ritz and Sejnowski, 1997; Van Vreeswijk et al., 1994). Especially for the biologically relevant case of non-instantaneous coupling and heterogeneous networks, the recurrent inhibition between excitatory and inhibitory sub networks was found to stabilize and induce zero phase synchronization reliably and quickly (Kopell et al., 2000; Van Vreeswijk et al., 1994). In addition, synchronization via recurrent inhibition can synchronize neuronal activity without changing the overall firing rate of the neurons (Tiesinga and Sejnowski, 2004; Buia and Tiesinga, 2006), as it was reported by many experimental studies. In contrast, instantaneous excitatory coupling was shown to favor desynchronization rather than synchrony for a broad class of models and parameter regimes (Kopell et al., 2000; Ritz and Sejnowski, 1997).

Compared to chemical coupling, the gap junction coupling is electrical and almost instantaneous. Its importance for generating oscillatory and synchronous activity was highlighted in experimental studies that demonstrated that fast oscillations can be observed even if chemical synaptic transmission is blocked (Buzsaki and Draguhn, 2004). The nature of gap junction coupling is diffusive and essentially homogenizes the membrane potential fluctuations in the network. This homogenization was shown to lead to complementary effects of electrical and chemical coupling for networks that comprises both types at the same time. It was shown that electrical and chemical coupling act similarly and add up linearly when the 
coupling of both types is weak. However, due to homogenization of fluctuations of the membrane potential, even weak gap junction coupling can boost the effect of the much stronger chemical coupling (Kopell and Ermentrout, 2004). This suggests that more than a single mechanism may contribute to zero phase synchronization and demonstrates that modifications of individual mechanisms can substantially alter the synchronization patterns.

Another class of mechanisms that support zero phase synchrony is based on spikes in rapid succession, e.g. doublets of spikes (Kopell et al., 2000). The model was confirmed by experimental findings in rat hippocampus slices, which linked the firing of spike doublets by interneurons to gamma oscillations that are synchronized over several millimeters (Traub et al., 1996). However, for long range synchronization across different regions and especially across hemispheres, none of the above mentioned local mechanisms is general enough. Synchronization via spike doublets breaks down for gamma frequencies when conduction delays become large and recurrent inhibition with or without gap junction coupling is only plausible for local networks due to the topology that is dominated by local neuronal coupling.

In addition, the topology of complex networks turns out to be another very important parameter for the establishment of local and long-range synchronization. Entrainment via an auxiliary hub can be seen as an extreme case (Huang and Pipa, 2007) but topologies also play an essential role in cases of emergent synchrony. Thus, hubs in the network were proposed to shorten the path length between elements and therefore to foster fast and stable synchrony (Arecchi et al., 1999; Grinstein and Linsker, 2005). An alternative approach to study dynamical properties and to infer functionality of the topology of complex networks is the decomposition of a network in principle building blocks, i.e. motifs that are repeated connectivity patterns within the network (Milo et al., 2002; Sporns and Kotter, 2004). However, a generalizing framework that describes synchrony in complex networks has not been found yet.

Finally, several authors have suggested that the reciprocal coupling of cortical areas with the different thalamic nuclei may support the coordination of distributed cortical processing (Llinas and Pare, 1998; Sherman and Guillery, 2002). The thalamus with its bidirectional and radial connectivity to the neo-cortex represents a simple network motif. It consists of a V-shaped configuration composed of three populations, two of which are bidirectionally coupled with the third. The special role of this third central population is that it redistributes common activity to the two other populations in a symmetrical way (Fischer et al., 2006). We could recently demonstrate that the dynamics in this network motif can naturally induce and stabilize zero phase lag synchronization between the outer populations for any conduction delay between the populations (Vicente et al., 2008).

The principle behind this mechanism is that relaying the activity of the outer two neuronal populations via a central mediator redistributes identical information to both outer elements and therefore allows for self-organized lag-free synchronization among the outer pools (see Figure 1). It is important to emphasize that the central element is the key for the communication among the populations, but does not dictate the dynamics as in the case of a master-slave system. Therefore, zero phase synchronization via relaying is an emergent property of all three populations. Remarkably, dynamical relaying has also been demonstrated to be an effective mechanism for inducing and stabilizing zero time lag synchronization between chaotic elements (Fischer et al., 2006). Regarding the temporal precision and the time the system needs to synchronize, the mechanism proved to be compatible with experimentally observed millisecond precise synchronization that is established within tens of milliseconds or very few oscillation cycles. The mechanism also overcomes the problems posed by long axonal delays and high frequencies that cannot be coped with by other synchronizing mechanisms. Thus, dynamical relaying based synchronization might provide a general solution for the establishment of long range neuronal synchrony, irrespective of whether the V-motif is implemented via corticothalamic or purely cortico-cortical connections.

\section{Near zero-phase synchronization}

In addition to zero-phase synchronization, oscillations also display synchronization with consistent phase differences of fractions of an oscillation cycle (see below). A special case of near zero phase synchrony are delays that are additive. In this case the relative phase difference between pairs of neurons can also be expressed as a relative delay compared to a single global synchronized dynamics of the network.

One possibility to explain near zero phase synchrony is detuning. In heterogeneous networks individual elements can have different intrinsic properties such as the resonant frequencies. When coupling of the intrinsically different elements leads to globally synchronized activity, the activity of each individual element needs to be adjusted to match global dynamics. For systems that are composed of oscillatory or excitable elements with different resonant frequencies it is well known that the adaptation to a single and global rhythm requires individual adjustments of phase advances and lags (Pikovsky et al., 2001; Schuster and Wagner, 1989). Therefore, detuning naturally leads to phase differences among the elements of the network. For oscillatory activity and global synchronization detuning even leads to additive phase differences, that can be expressed for each individual as a phase difference to a single global dynamics. Additive phase relations were also discussed to be caused by small differences in conduction delays from a common drive. Regarding the encoding of information (see 'Novel Empirical Results on Temporal Coding'), both the detuning but also the common drive hypothesis open the possibility to encode information in the relative timing of the discharges of individual neurons through a temporal code that is based on phase relations.

\section{NOVEL EMPIRICAL RESULTS ON TEMPORAL CODING Function of near zero phase lags in synchrony}

The early work on synchronized cortical events indicated that many of these phenomena do not result from stimulus locked synchronization but from internally generated temporal patterning based on gamma and beta oscillatory activity. Meanwhile, it became apparent that systematic phase-lags occurring with sub-millisecond precision may enable the cortex to exploit temporal relationship between action potentials for information coding in sensory systems.

A coding mechanism based on phase lags has been already discovered in the hippocampus and is supported by the internally generated oscillations in the theta range $(5-8 \mathrm{~Hz}$ ) (O'Keefe and Recce, 1993) and in the neo-cortex for oscillations in the gamma range $(>40 \mathrm{~Hz})$. These oscillations are not locked to the stimuli but 
A

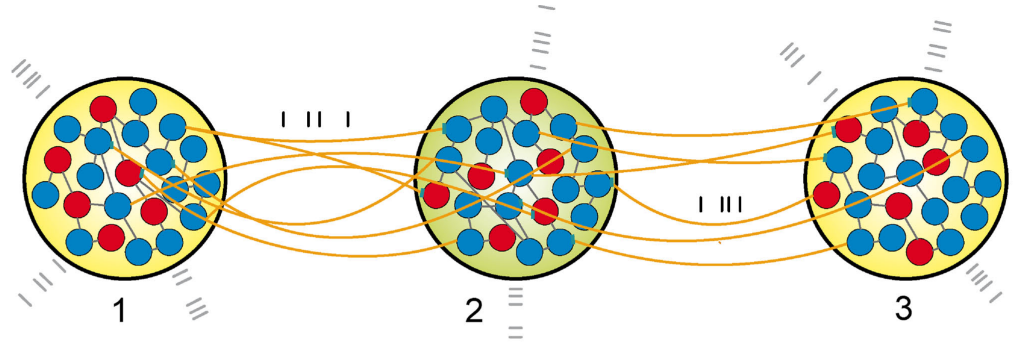

B
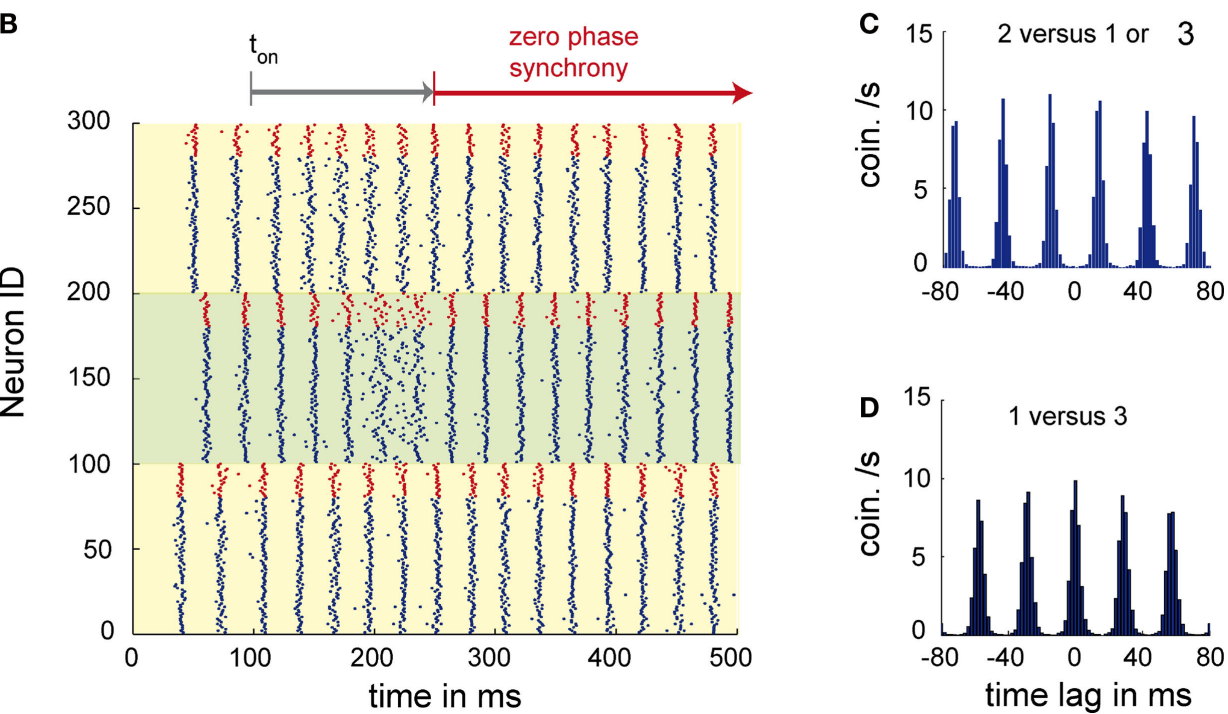

FIGURE 1 | Dynamics of three networks of excitatory and inhibitory neurons interacting through dynamical relaying. (A) Topology of the relaying motif. Here, three networks 1, 2, 3 each composed of 20 inhibitory (red) and 80 excitatory (blue) neurons. To control the activity level of each population external Poisson input is used (grey spikes). The conductions delays are $12 \mathrm{~ms}$ and therefore significant compared to the period of the oscillation. (B) Raster plot of the activity of each neuron of population 1 (neurons 1-100), population 2 (neurons 101-200) and population 3 (neurons 201-300). Blue spikes are from the excitatory and red spike from the inhibitory neurons. At coupling $t_{\text {on }}$ at $100 \mathrm{~ms}$ the coupling between the populations was activated. After 90 ms of activated coupling P1 and P3 start being zero-phase synchronized. The whole synchronization period lasts about 130 ms. Note that the activity of the relaying population P2, gets less synchronized due to individual phase adaptations of P2 neurons during the synchronization period between 160 and $230 \mathrm{~ms}$. This indicates that the relaying population $\mathrm{P} 2$ is not acting as a master that entrains $\mathrm{P} 1$ and $\mathrm{P} 3$, but that synchronization is established between $\mathrm{P} 1$ and $\mathrm{P} 3$ via relaying of activity through P2. (C) Average cross-correlation between population P1 or P3 and the relaying population P2. (D) Average cross correlation between the outer populations P1 and P3. are generated by internal mechanisms independently of stimulus timing. The neurons discharge during particular phases of the oscillation cycle whereby the relative phase of spike generation varies in a context dependent way. In the hippocampus cells fire progressively earlier in the theta-cycle as the animal traverses the cell's place field, a phenomenon called phase precession (Buzsaki, 2002; O'Keefe and Recce, 1993). Consequently, neurons that follow the same rhythm generate also action potentials in precise temporal relationships relative to each other. Oscillations in the theta frequency range are also observed in the neocortex where they coexist with much faster oscillations in the beta and gamma bands, i.e. $>\sim 20 \mathrm{~Hz}$.

These high frequency oscillations are important for the generation of precise temporal relations and neuronal synchrony. Two neurons are said to synchronize their discharges when the likelihood that they will both fire action potentials within a small time window (up to several milliseconds wide) is much higher than what is expected by chance. The chance level is determined from the overall firing rates of the neurons. For a pair of neurons, synchronization is commonly assessed from the narrow centre-peaks in cross-correlation histograms (CCH) (see example in Figure 2A) but recently also methods have been developed that can detect synchrony simultaneously across a large number of neurons (Pipa et al., 2007, 2008). These methods overcome certain limitations of pair-wise $\mathrm{CCH}$ analysis and permit rate corrected detection of higher order correlations.

There are two ways how neuronal synchrony may support the encoding of information about stimuli. The first and the most extensively investigated is the modulation of the strength of synchronization. The degree to which neurons synchronize their discharges can vary and depends on many variables, such as the similarity of the feature preferences of neurons and the spatial distance between them. Neurons in the visual cortex are more likely to synchronize their responses the more their orientation preferences are similar and the closer their receptive fields are in space (Betsch et al., 2004). These and other results (Gray et al., 1989) have led to the hypothesis that the strength of neuronal synchrony serves to signal information 


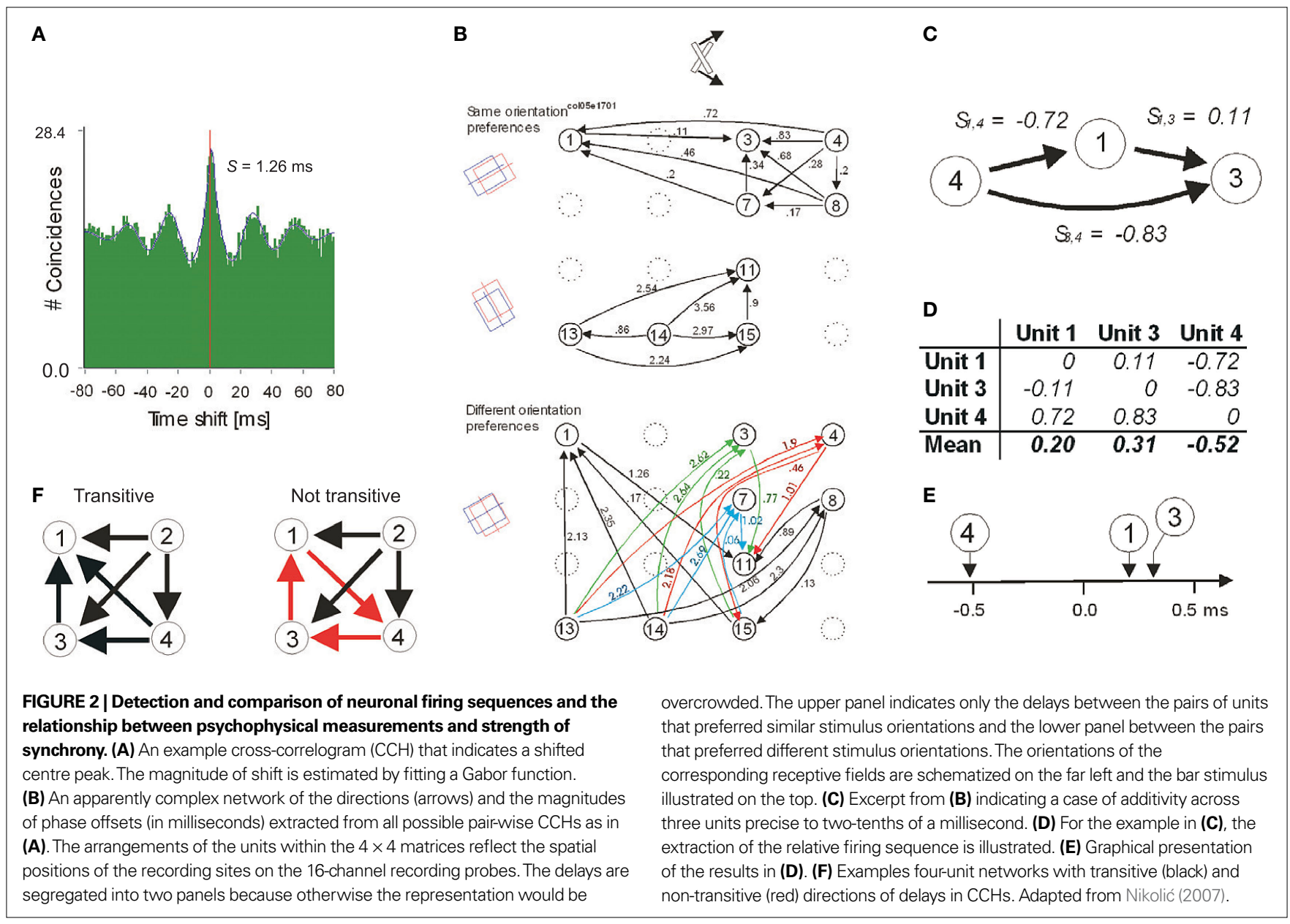

about the relatedness between the visual features that activate those neurons (Singer, 1999).

The second principle for coding information by neuronal synchrony is similar to that in hippocampus and utilizes the delays or phase-offsets among the discharges of neurons that are participating in synchronized assemblies. In the neocortex, neurons are rarely synchronized with exact zero delays. Instead, CCHs usually reveal small but highly stable and significant delays (Havenith et al., 2009; König et al., 1995; Nikolić, 2007; Roelfsema et al., 1997; Schneider and Nikolić, 2006). These delays may extend up to $\sim 15 \mathrm{~ms}$ (Schneider and Nikolić, 2006) and do not reflect conduction delays as they change as a function of stimulus properties. They also vary systematically for different pairs of simultaneously recorded units, which allows one to extract a one-dimensional representation of the relative firing times for all coupled, i.e. synchronized neurons (Figures 2B-E). Thus, cortical neurons exhibit systematic differences in their firing times relative to some internal reference. Systematicity can be formally conceived as additivity of phase delays (e.g., Figure 2C), which allows parametric analysis based on ANOVA (Schneider et al., 2006). Alternatively, the directions of phase offsets can be investigated without considering their magnitudes in which case they are treated as mathematical transitivity and can be subjected to non-parametric analysis (Figure 2F) (Nikolić, 2007).
Unpublished observations support the notion that the internal reference signal for the rank ordering of phase offsets are the-often stimulus induced-oscillations in the high frequency range (beta and gamma). Most importantly, these delays, and thus also the relative firing times, change dynamically as a function of stimulus properties (König et al., 1995; Nikolić, 2007; Schneider et al., 2006). This led to the hypothesis that the relative time at which a neuron sends action potentials could be exploited to encode stimulus-related information (Nikolić et al., 2004). One possible encoding principle is a conversion of an amplitude code to a temporal code, such that more strongly depolarized neurons generate action potentials earlier than the less optimally stimulated ones (Fries et al., 2007).

In Figure 3A we show a firing sequence determined from the spike-trains obtained from 14 units in area 17 of a cat. Figure 3B indicates that this sequence is reliable when the same stimuli are presented twice and Figure 3C that the sequence changes if stimulus properties are changed. The extent to which this putative code can be used for computations in the brain and the accuracy with which it can carry information are yet to be investigated. Our results suggest that the trial-to-trial variability of firing sequences can be comparable to that of neurons' firing rates, the pre-condition for such a remarkable coding precision being the presence of strong beta/gamma oscillations (Havenith et al., 2007). 
In future, the spatial extent to which neurons can engage into this distributed code and its possible specificity for certain cortical layers or for given cortical areas will need to be determined. There are several options how this information can be read out by individual neurons. Simple integrate-and-fire neurons with sufficiently short integration time constants have been shown to be sensitive to information encoded in the precise stimulus-induced temporal relations of neuronal discharges (Nikolić et al., 2007). It remains to be seen how these properties and the implementation of additional computational capabilities through active dendritic mechanisms improve the readout of the rich temporal relations resulting from internal patterning of response timing. The available evidence suggests that the biophysical properties of the dendritic processes are well suited for such readout of coincident events (Williams and Atkinson, 2008) and temporally ordered information. The exquisite sensitivity of spike timing dependent plasticity (STDP) for the sequence order of pre- and post-synaptic activity indicates that precise temporal relations matter in neuronal processing and can be read out efficiently (Markram et al., 1997).

The main motivation for the present studies is that the firing sequences driven by beta/gamma activity and defined within a single oscillation cycle may constitute a mechanism for the encoding and exchange of information in the cortex. Thus, in addition to the firing rates, precise timing of individual discharges may be used to gate transmission and synaptic plasticity, to selectively route activity across the densely interconnected cortical network and to define particular relations in distributed activity patterns. This hypothesis is directly related to the present research and needs to be pursued. If supported by further data it will have profound implications for our understanding of brain function. Temporal sequences may be

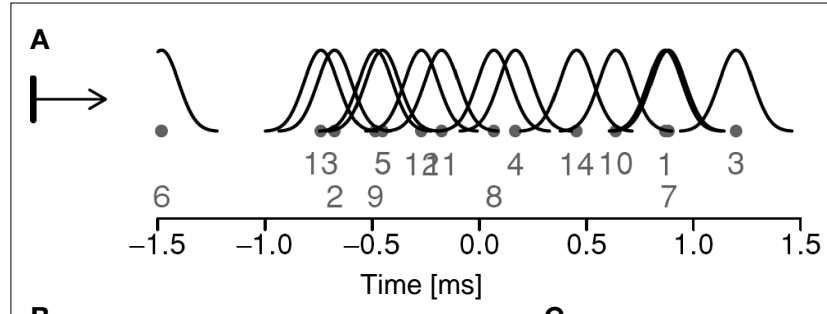

B
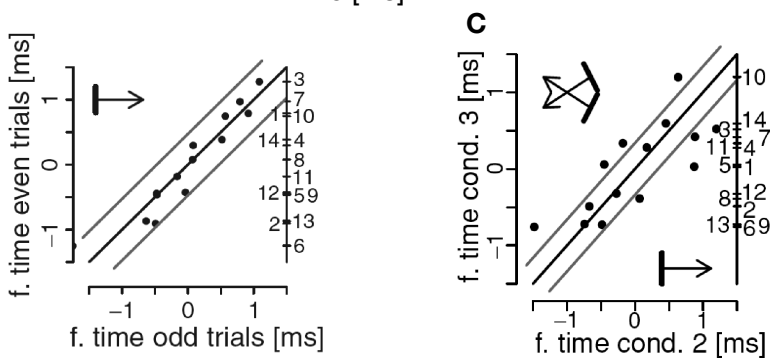

FIGURE 3 | (A) A firing sequence extracted for a network of 14 units. The dots denote the estimated positions of the units, the number their identities, and the black curves indicate the localization errors. (B) The relative firing sequence in (A) estimated for two repetitions of the same stimuli. Gray lines parallel to the diagonal indicate error limits of two standard deviations. Unit identities are indicated on the right side of the panel. (C) Changes in firing sequences as a function of a change in stimulus properties. Stimuli are sketched in the corners of the graph. Units outside the error lines change significantly their preferred firing times. Adapted from Schneider et al. (2006). a fundamental mechanism by which the nervous system achieves its function. Likewise, beta/gamma oscillations could serve not only to adjust the timing of action potentials but they could also provide an internal temporal reference for the extraction of this information.

\section{Complementary rate and temporal code}

In addition to studying putative principles of temporal coding, it is necessary to collect evidence for a functional role of synchrony. One indication comes from a study in which we related changes in synchrony to a perceptual phenomenon called apparent brightness (Biederlack et al., 2006). With multisite recordings in the cat visual cortex we compared directly the changes in discharge rate and synchrony with stimulus modifications that lead to changes in apparent brightness. This revealed a close match between a psychophysical function describing the magnitude of the visual illusion on the one hand, and the change in the strength of synchronization between the neurons responding to the stimulus, on the other (Figures 4A,B). Moreover, by comparing changes in discharge rate and synchrony for different stimulus configurations the study indicated that an

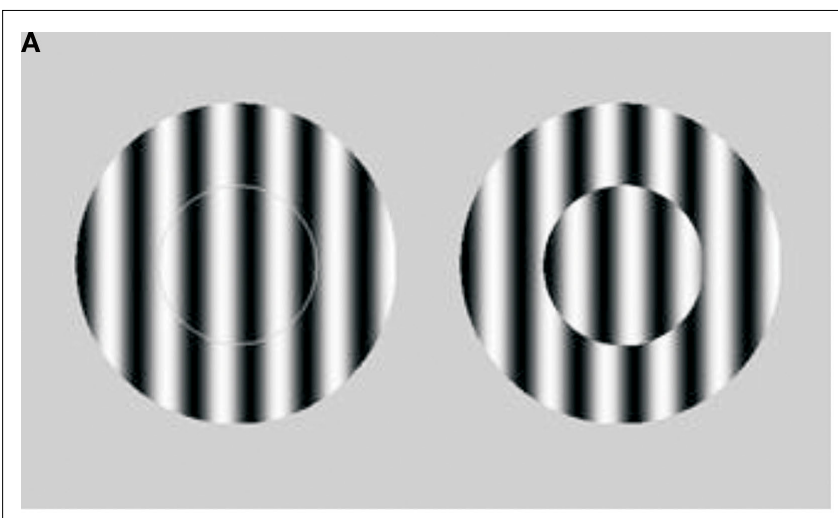

B

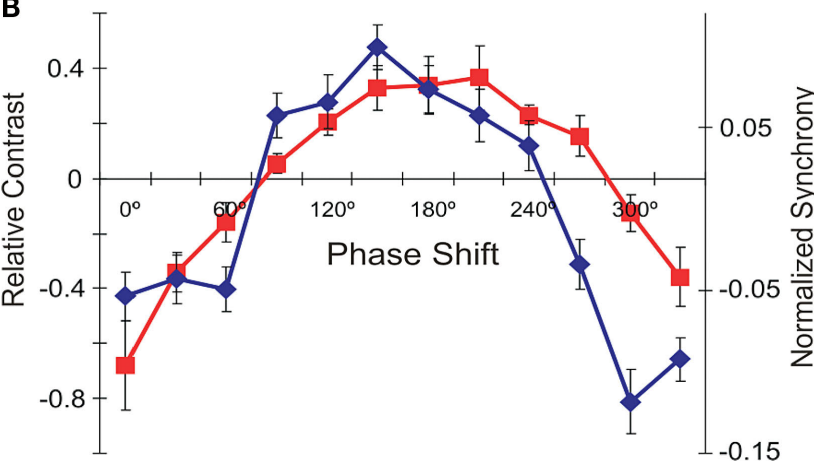

FIGURE 4 |The role of synchrony for perception of brightness. (A) In the two centre-surround stimuli, the gratings in the centre are physical identical. Nevertheless, most observers report seeing stronger contrast on the right where the centre is offset for $180^{\circ}$ relative to the surround. (B) Experimental results obtained with stimuli shown in (A). Red: Human psychophysical judgments of changes in perceived contrast as a function of phase offset between the centre and surround. Blue: Changes in the strength of synchronization between neurons in cat area 17 whose receptive fields were covered by the centre stimuli and whose orientation preferences matched the orientation of the grating. Adapted from Biederlack et al. (2006). 
increase in synchrony produces a perceptual effect that is equivalent to an increase in firing rates. That is, if neurons synchronize more strongly, the stimulus is perceived more intensively. The significance of this study is that conclusions are based on a close match between complete physiological and psychological functions, which were determined for the entire spectrum of stimulus properties, exceeding thus the usual correlative evidence based only on extreme values (e.g., minimum and maximum stimulus intensities).

This type of analysis allows one to falsify theories even when the analyses of extreme values support them and are correlated perfectly. In our case, this allowed us to falsify an alternative hypothesis that synchrony accounted for figure-ground segregation, which, besides the extremes, showed otherwise completely uncorrelated measures (Biederlack et al., 2006). At the same time, by showing a close match between increased perceived brightness and either increased synchrony or increased discharge rate, the results showed the perceptual equivalence of raising the saliency of responses either by increasing discharge rate or synchrony.

\section{CONCLUDING REMARKS}

In conclusion, in this section we have first presented a theoretical framework for synchronization among neurons with delays smaller than the conduction delays. Next, we have reviewed empirical evidence for the existence of such delays and for their functional role in brain computations. These studies are at the very beginning of the search for temporal codes and we expect the research in this direction to expand. It will be a great challenge to provide direct causal proof that changes in firing sequences affect behavior or psychological experience (e.g., perception). However, this applies equally to all other codes that have been investigated in the attempt to bridge the brain-mind gap.

\section{BUILDING EXPECTATIONS: NEW VISTAS FOR GAMMA OSCILLATIONS}

As argued above, a critical step in understanding perceptual organization is to find out how the brain builds stable relationships among sensory inputs. For this, however, it is not only important to know about encoding principles, but also to probe ongoing states of expectancy and attention. Beyond a purely sensory-driven approach to perception, this view focuses on top-down mechanisms which are related to large-scale dynamics at multiple scales. The mechanisms underlying these processes are still largely unknown. As discussed before, an attractive hypothesis is that precise temporal patterning of neuronal activity provides a framework for contextual links (Engel et al., 2001; Singer, 1999). In this model, sensory inputs interact with internally generated signals necessary for building predictions about the world.

Evidence in support of the neuronal synchronization hypothesis was initially obtained in anesthetized animals, and later extended to waking and conscious states, as described in cats (Gray and Viana Di Prisco, 1997; Roelfsema et al., 1997; von Stein et al., 2000), nonhuman (Eckhorn et al., 1993; Frien and Eckhorn, 2000; Gail et al., 2000; Kreiter and Singer, 1992, 1996; Maldonado et al., 2000) and human primates (Palva and Palva, 2007; Rodriguez et al., 1999; Tallon-Baudry et al., 1997). Notwithstanding these findings, after two decades of research, the role of gamma in feature binding remains a matter of dispute and controversy (Ghose and Maunsell,
1999; Roelfsema, 2006; Shadlen and Movshon, 1999; Singer, 1999). In monkeys, several studies designed to test for perceptual grouping failed to support the synchronization hypothesis (Dong et al., 2008; Lamme and Spekreijse, 1998; Palanca and DeAngelis, 2005; Roelfsema et al., 2004; Thiele and Stoner, 2003).

More recently, however, evidence became available that synchronization of gamma oscillatory responses are closely linked to more general cognitive functions such as selective attention (Doesburg et al., 2008; Dong et al., 2008; Fan et al., 2007; Fries et al., 2001a; Lakatos et al., 2008; Roy et al., 2007; Steinmetz et al., 2000; Taylor et al., 2005; Womelsdorf and Fries, 2006; Womelsdorf et al., 2007), short- and long-term memory (Herrmann et al., 2004; Pesaran et al., 2002; Wu et al., 2008) and multisensory integration (Lakatos et al., 2007), suggesting that gamma-oscillations may have a much wider relevance in cortical networks for behavioral and cognitive phenomena.

In this section, we present new experimental evidence supporting this hypothesis by showing that, in addition to selective attention, preparation to respond or perceptual readiness also leads to a selective increase in the gamma components of the responses. Since the modulation in gamma power associated with readiness exhibits very fast dynamics, it may play a particularly important role in perceptual organization, as briefly discussed below.

It is well established that arousal and attention are of general relevance for visual processing (Posner, 1980). Studies in monkeys and humans have shown that attention can selectively enhance processing of image features (Desimone and Duncan, 1995; Reynolds and Desimone, 2000; Sheinberg and Logothetis, 2001; Spitzer et al., 1988). Directing attention to a stimulus at a particular location leads to an increase in its effective contrast (Carrasco et al., 2004; Reynolds and Chelazzi, 2004) and can modulate tuning properties (Roberts et al., 2007; Spitzer et al., 1988). Given the central role of attention in perception, Fries and collaborators, decided to investigate whether gamma oscillations could also serve as a selection mechanism. In recordings from area V4, they have found strong phase-locking between spiking activity and ongoing LFP oscillations confined to the gamma band (Fries et al., 2001a, 2008; Womelsdorf et al., 2006, 2007). Based on these findings, a mechanism was proposed in which effective communication within distributed neuronal networks relies essentially on the precise temporal relationships (Fries, 2005; Fries et al., 2007, 2008). According to this hypothesis, attentional enhancement of neuronal synchronization would facilitate selectively the communication among synchronized assemblies, which is thought to be important for a large number of operations underlying cortical processing (Azouz and Gray, 2003; Salinas and Sejnowski, 2000; Sejnowski and Paulsen, 2006).

In order to investigate the dependency of gamma oscillations on attentional preparation, or readiness, we have trained macaque monkeys to respond to a variety of stimuli, such as gratings, plaids and Gabor patches (Lima et al., 2006, 2007; Neuenschwander et al., 2008). In all these paradigms the monkeys were required to detect a stimulus change at fixed time points within the trial. Here we present results obtained for responses to Gestalt figures embedded in 2D-arrays of Gabor patch elements (Neuenschwander et al., 2008). During this task, the Gabor elements changed their orientation randomly every $400 \mathrm{~ms}$. At a fixed time point (3200 ms), a subset of neighboring Gabors were aligned to form a closed 
figure. The appearance of the figure required a motor response, and therefore, implicitly carried a behavioral relevance. Thus, in anticipation of the stimulus, the monkeys were likely to build up expectancy, or response readiness along the course of the trial.

Neuronal responses were recorded at multiple sites in area V1. The embedded figures were constructed as a function of the position and properties of the receptive fields of the neurons. In this way, a unique figure was designed for every new set of recording sites. Within the trial, the changes in the orientation of the Gabor elements were set to match the preference of the cells at fixed epochs, coinciding or not with the appearance of the figure. Thus, we could study the same local responses in two contexts: in one case associated with a global percept (Figure 5), and in the other not. Figure 5A shows the average peri-stimulus time histogram (PSTH) obtained for a single cell. Robust responses to the matching
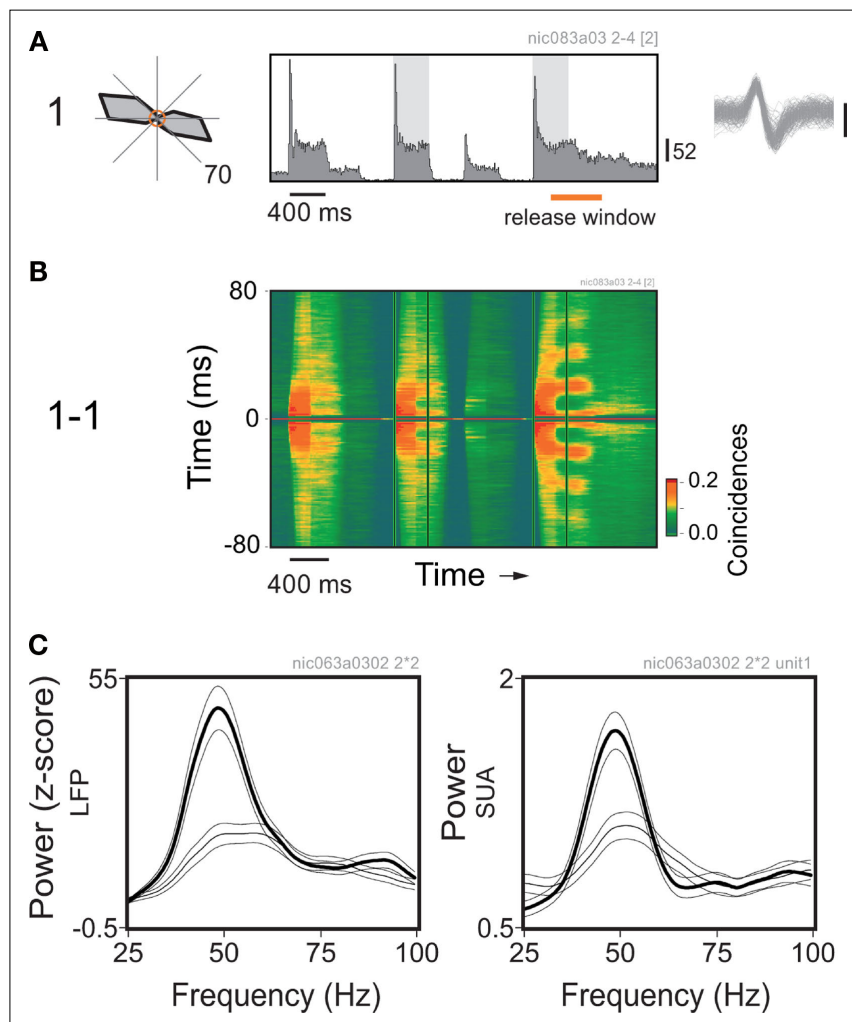

FIGURE 5 | Perceptual readiness modulates gamma power in V1 of the monkey. (A) Average response histogram for a single cell to a Gabor element centered over its receptive field. The orientation of the Gabor element changes at $400 \mathrm{~ms}$ steps. Notice the strong responses for the epochs of orientation match. Analysis windows are indicated by the shadowed rectangles on the plot. Direction tuning is shown on the left. Spontaneous activity is represented by the central circle, maximum average rate is indicated at the bottom right of the plot. (B) Sliding window autocorrelation for the responses shown above. Plots are aligned in time. Autocorrelation functions were computed for $200 \mathrm{~ms}$ windows at steps of $50 \mathrm{~ms}$. (C) Corresponding power spectra obtained for the local field potential (LFP) and spiking activity recorded from the same electrode. Thin line: window of match in the middle of the trial. Thick line: window of match prior to the behavioral response (in this example, coincides with the appearance of the embedded figure). Multitaper spectral analysis was obtained using the Chronux analysis software. Power was normalized by the firing rate. Thin lines around the mean indicate $95 \%$ confidence intervals. orientation of the Gabor element are readily visible in the histogram as brief transient peaks followed by a sustained component. The last epoch of orientation match was the one associated with the figure. At this very moment the monkey was required to respond within $500 \mathrm{~ms}$ with a lever release. Notice that response levels were similar for all epochs in which the stimulus orientation matched the orientation preference of the neurons.

A dramatic difference, however, was revealed in the temporal structure of the responses. As shown by the sliding autocorrelation analysis of the spiking activity (Figure 5B), there was a marked increase of gamma oscillatory components developed prior to the behavioral response. Accordingly, the spectral analysis of the LFP and single cell spiking activity showed a peak at around $50 \mathrm{~Hz}$, which was more pronounced for the window associated with the behavioral response (Figure 5C). Controls were also made for behavioral responses without the appearance of an embedded figure (not shown). In those cases the monkey had to report a color change of the fixation point. Surprisingly, we have found no significant differences in gamma modulation between the conditions with and without the embedded figure, indicating that the stimulus per se was not responsible for the enhancement. Thus, there was no simple relation between gamma synchronization and the binding of elements into a figure.

Taken together, our results suggest that gamma activity is strongly modulated by central states, probably related to perceptual readiness or readiness to act. A potential explanation could be higher levels of arousal associated with the anticipation of behavioral responses. States of central activation are associated with cholinergic facilitation of gamma activity (Herculano-Houzel et al., 1999; Rodriguez et al., 2004). It remains to be clarified, however, whether there is a direct link between the motor response preparation per se and the modulation of gamma activity in V1. The fast modulation of gamma power in the present experiments could be mediated by top-down influences or ascending cholinergic mechanisms controlling the intrinsic dynamics of the cortical networks. In either case, the anticipatory entrainment of the visual cortex in highly synchronous gamma oscillations is likely to be instrumental for the expected processing of sensory signals and their propagation towards executive structures. Recently it has been shown that coherence between motor cortex and spinal motor neurons increases as function of readiness to respond (Schoffelen et al., 2005), similar to our results in V1. These corticospinal interactions haven been interpreted as evidence for a role of gamma synchronization in neuronal communication (Fries, 2005). It would be interesting to see, whether more anterior cortical regions also engage in anticipatory gamma activity and whether there are any consistent temporal relationships.

\section{NEURAL SYNCHRONY AS A MECHANISM FOR CONSCIOUS PERCEPTION?}

The distributed organization of the primate brain poses particular challenges for understanding the integrative aspect of cognition, of which consciousness is the utmost example. The intentional' feature of consciousness, the fact that our experience always refers to something, suggests that understanding how the content of cognition is represented in the brain, and how unified experience can emerge out of distributed brain activity might be particularly informative 
in the quest for the neuronal correlates of consciousness (NCC) ${ }^{1}$. Thus, theories of binding and large-scale integration have recently come into the focus of the research on consciousness (Engel and Singer, 2001; Thompson and Varela, 2001).

One candidate mechanism for dynamic sub-system integration is the precise synchronization between the participating neuronal assemblies (Varela et al., 2001). Several properties make neuronal synchrony worth considering in the context of the NCC. Besides offering a mechanism to link elementary features together and thus allowing for the representation of composite perceptual objects, binding by neuronal synchrony could confer the brain with a vast and economical representational space (Singer, 2002).Considered at a large scale, synchronization of distant neuronal assemblies could allow for subsystem integration and thus account for the unified aspect of conscious experience (e.g., where different sources of information are bound in an integrative cognitive moment). However, processes explaining how the unity of conscious experience is achieved do not suffice alone as a potential mechanism for consciousness. Any given theory of the NCC should also at least explain how different percepts dynamically map into different states, and how the system dynamically selects subsets of neuronal responses for conscious representation. Importantly, given that a considerable amount of cognitive and executive operations can be performed without being aware of them (Dehaene et al., 1998, 2001; Sergent et al., 2005), it is also necessary to distinguish between these two types of processing (conscious and non-conscious) at the neural level. Evidence suggests that changes in neuronal synchrony could account for these two processing modes. First, regular changes in the content of consciousness can be represented when considering that groups of neurons can take part or drop off from the dynamically linked network. The formation of assemblies would be only limited by the time needed to establish stable states between them. This property could then account for the transitoriness and temporal flow of experience (Varela, 1999). Second, due to the coincidence sensitivity of cortical neurons synchronized inputs enhance the probability of response in target neurons (Salinas and Sejnowski, 2001), and this in turn would allow for the amplification of neuronal responses and selection of subpopulations of responses based on the strength and propagation of their activity (Jensen et al., 2007). Third, the required switch between conscious and unconscious processing could be achieved by varying the spatial scale of the synchronized neuronal assemblies. Conscious cognition would involve long-distance coordination between distributed neuronal groups, whereas unconscious cognition would be instantiated by local coordination (Dehaene et al., 2006; Thompson and Varela, 2001). Small world networks, of which the brain seems to be one example (Yu et al., 2008), combine these two processing modes: local modularity and long-range connectivity. Thus, depending on dynamic shifts in coupling, the same anatomical network can support both local and global - or subconscious and conscious - operations (Buzsaki, 2007).

${ }^{1}$ In the context of this review consciousness will be understood as the subjects' ability to detect (report the presence or absence) or identity (discrimination) a particular stimulus. In contrast, failure to detect or to identify will be taken as indication of unawareness. Thus, awareness refers to the representation of internal or sensory changes that are accessible to introspection or direct report. An NCC will be defined as the minimal set of neuronal events that are necessary and/or sufficient for perceptual awareness (Chalmers, 2000; Kanwisher, 2001).
Long range synchronization in the beta and gamma range has been observed with scalp as well as with intracranial recordings when a coherent and unified percept emerges in response to a given stimulus (Lutz et al., 2002; Rodriguez et al., 1999; Sehatpour et al., 2008). For instance, Sehatpour et al. (2008), using intracraneal electrodes, reported robust coherence in the beta-band between the hippocampal formation, occipitotemporal cortex, and lateral prefrontal cortex when participants recognized fragmented images. In contrast, when scrambled versions of the same images were presented significantly lower coherence was observed. These results suggest that transient oscillatory coupling between distributed cortical regions may underlie effective communication during visual object processing. Furthermore, in conditions of competition such as in binocular rivalry selection of responses for further processing appears to be achieved via increases in neuronal synchronization of groups of neurons in the primary visual cortex of the cat (Fries et al., 1997, 2002), while discharge rate of neurons does not distinguish between the dominant (perceived) and non-dominant (suppressed) pattern. Studies employing MEG and EEG in humans yielded similar results (Cosmelli et al., 2004; Doesburg et al., 2005; Srinivasan et al., 1999; Tononi et al., 1998): Perceived stimuli correlate with increased synchronization in the gamma range between distal cerebral areas (occipital and frontal regions) (Cosmelli et al., 2004). Similar results have been obtained in the attentional blink paradigm. Successful detection of stimuli was accompanied by increased synchronization in the beta range. Source analysis revealed, as in the case of binocular rivalry, dynamical coordination in the beta range between frontal, parietal, and temporal regions for detected targets (Gross et al., 2004).

Recently, in order to directly explore the relation between neuronal synchrony and conscious perception, we designed a paradigm in which the electrophysiological signatures related to conscious and unconscious processing of visual stimuli were directly compared (Melloni et al., 2007). Subjects had to detect and identify a briefly exposed word $(33 \mathrm{~ms})$ presented between masking stimuli. The strength of the masks was adjusted such that in half of the trials subjects had no conscious recollection of having seen the word. After a delay of $\sim 500 \mathrm{~ms}$ a second word was presented, always visible, and the subject had to decide whether the two words were the same or different. Importantly, in an attempt to study the processes related to conscious perception dissociated from other cognitive processes such as decision making and motor response, the first stimulus over which visibility was manipulated was separated in time from a second stimuli over which subject had to respond.

To study the neuronal signatures related to conscious processing and how they evolve over time we simultaneously recorded electroencephalographic activity (EEG) and subsequently analyzed different measures of neuronal responses: time resolved power changes of local signals, the precision of phase synchronization across recording sites over a wide frequency range, and event-related potentials. The first electrophysiological event that distinguished seen from unseen words was an early and transient burst of long-distance synchronization observed in the gamma frequency. Regional distributions of gamma spectral power and phase revealed that the amplitude and patterns of gamma oscillations were spatially homogeneous and similar for the two conditions, while the patterns of phase synchrony were markedly different (Figure 6). Significant 


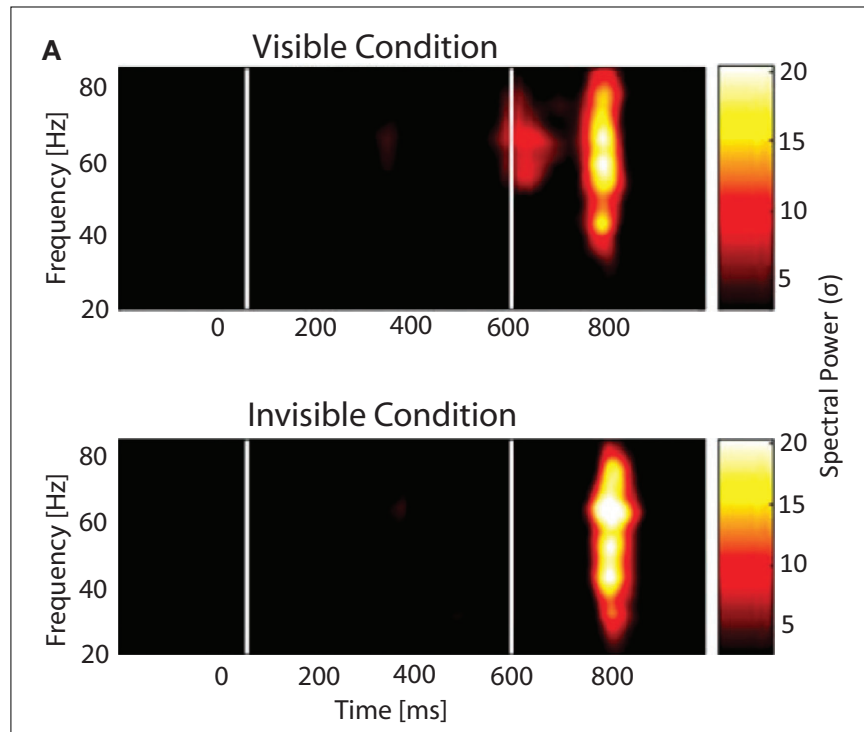

FIGURE 6 | Spectral power and phase synchrony to visible and invisible words. Words were flashed for $33 \mathrm{~ms}$ and surrounded by masks that depending on their luminance rendered the stimuli either visible or invisible. After around $500 \mathrm{~ms}$ a second word was presented and subjects had to determine whether it was the same or different from the previously presented word. (A) Time-frequency plot and (B) Phase synchrony plot shows the grand average for

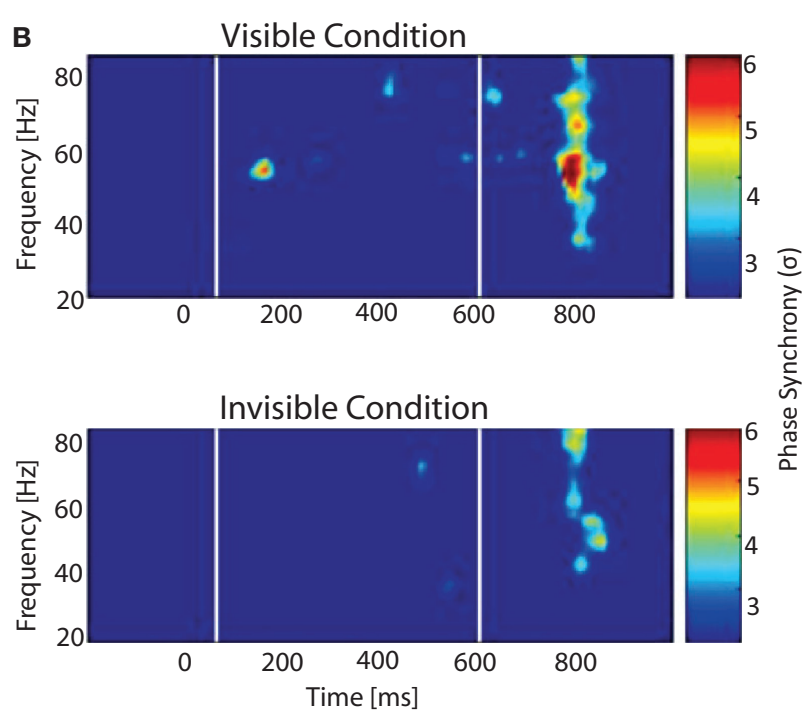

all the electrode pairs. White lines depict the time when the first and second word were presented. Color scale corresponds to amplitude expressed in SD, calculated over a 500-ms baseline. (A)Time-frequency plot: Two increments in gamma oscillations are visible. The first, only present in the visible condition, the second in both conditions. (B) Phase locked synchrony: The first peak is only present during the visible condition, whereas the second one is observed for both conditions. phase locking within and across hemispheres, between occipital, parietal and frontal site was observed only for the visible condition and was absent in the invisible condition.

After this transient period of synchronization, an increase in amplitude of the P300 component was observed, which has been interpreted as a correlate of the transfer of information into working memory. Furthermore, we observed increases in amplitude of theta oscillations over frontal electrodes during the interval in which visible words had to be kept in memory, in accordance with the assigned role of frontal theta oscillations in the maintenance of short term memories (Jensen and Tesche, 2002; Schack et al., 2005). The specificity of long-distance synchronization for conscious perception was further supported in an additional experiment controlling for the depth of processing (Melloni and Rodriguez, 2007). Altogether our results show that the precise phase locking across a widely distributed cortical network, and not the power of the local stimulus induced gamma oscillation, is the earliest signature of conscious processing. This suggests that conscious processing requires a particular dynamical state of the cortical network. We propose that this transient synchronization might enhance the saliency of the activation patterns not only allowing for contents to get access to consciousness but also triggering a cascade of processes such as perceptual stabilization, maintenance in working memory, and generation of expectations, all aspects intimately related with conscious awareness. It is at present unknown whether the early large-scale synchronization is already the neuronal correlate of phenomenal awareness or whether awareness emerges only from the entirety of the processes following this coordinated state. In addition, it remains to be clarified whether the large scale synchronization transfers contents into awareness and/or maintains them. The transient nature of the effect argues in favour of the first possibility.
The subsequent theta oscillations might then support maintenance. The rapid update of new contents might be instantiated by the short periods of long-distance synchronization in the gamma band, while the slower pace of theta oscillations might relate to sustained integration and maintenance of local results. The interplay between these two frequency bands might underlie the phenomenon of continuous but ever changing conscious experience.

\section{NEURAL SYNCHRONY IN SCHIZOPHRENIA AND AUTISM SPECTRUM DISORDERS}

In addition to the putative role of neural synchrony in temporal coding, top-down modulation of sensory signals and large-scale integration of distributed neural assemblies, there is increasing evidence suggesting that abnormal neural synchrony is a pathophysiological mechanism in neuropsychiatric disorders. Accordingly, in the following section, we will examine the evidence for a relationship between alterations in neural synchrony and the pathophysiology of schizophrenia and autism spectrum disorders (ASD) ${ }^{2}$. These results suggest that there are fundamental changes in neural synchrony that may underlie the cognitive dysfunctions associated with these disorders as well as certain symptoms.

Testing this hypothesis may have important implications both for the potential understanding and treatment of the disorders as these belong to the most costly public health problems. Furthermore, research into brain disorders also holds the potential to gain novel insights into the functional relevance of neural synchrony during normal brain functioning by establishing close correlations between

${ }^{2}$ Alterations in neural synchrony are not confined to these disorders (see Uhlhaas and Singer, 2006, for a review). Alterations in neural synchrony are also involved in Epilepsy, Parkinson's Disease and Alzheimer's Disease, for example. 
abnormal synchronization and specific cognitive deficits in a variety of neuropsychiatric disorders.

\section{SCHIZOPHRENIA}

From its earliest beginnings, the pathophysiology of schizophrenia has been described as a disorder involving a deficit in the integration and coordination of neural activity that leads to dysfunctions in cognition. Thus, Bleuler (1911) chose the word 'schizophrenia' to highlight the fragmentation of mental functions. Given the fundamental role of neural synchrony in establishing coherent mental contents (see 'Neural Synchrony as a Mechanism for Conscious Perception?' by Melloni), it can therefore be proposed that impaired neural synchrony underlies the fragmentation of mental functions in schizophrenia.

Indeed, current theories of schizophrenia (Friston, 1999; Phillips and Silverstein, 2003) converge on the notion that core aspects of the pathophysiology are due to deficits in the coordination of distributed neural processes that involve multiple cortical areas. This perspective contrasts with earlier views that emphasized a regionally specific pathophysiological process as the underlying cause for the signs and symptoms of schizophrenia. Some of the cognitive deficits in schizophrenia concern functions, such as working memory, attention, and perceptual organization, have been proposed to involve synchronization of oscillatory activity in the betaand gamma-band band (see Phillips and Silverstein, 2003, for a review).

A substantial body of EEG ${ }^{3} / M E G$-studies now support the hypothesis that cognitive deficits are related to impaired neural synchrony. Examination of auditory and visual steady-state responses to repetitive stimulation in patients with schizophrenia has revealed a specific reduction in the power of the stimulus-locked response in the beta- and gamma-frequency range, but not in the lower frequencies (Krishnan et al., 2005; Kwon et al., 1999). Reductions in evoked oscillatory activity have been reported for tasks involving visual binding (Spencer et al., 2003, 2004), for backward-masking (Wynn et al., 2005), in auditory oddball-paradigms (Gallinat et al., 2004) and during TMS-evoked activity over frontal regions (Ferrarelli et al., 2008). These results suggest selective deficiencies in the ability of cortical networks or cortico-thalamo-cortical loops to engage in precisely synchronized high-frequency oscillations.

Recently, we have tested the involvement of high-frequency oscillations with MEG in chronic-schizophrenia patients during the perception of Mooney faces (Tillmann et al., 2008) (see Figure 7). Mooney faces consist of degraded pictures of human faces where all shades of gray are removed, thus leaving only black and white contours. MEG signals were analysed for spectral changes in oscillatory activity in the frequency range of $25-200 \mathrm{~Hz}$. Compared to healthy controls, schizophrenia patients showed a highly significant reduction in high-frequency gamma-band activity $(60-120 \mathrm{~Hz})$ over parieto-occipital sensors which was accompanied by a significant deficit in the ability to detect upright Mooney faces. Thus, these findings suggest a comprehensive deficit in the regulation of

${ }^{3}$ Recently, it has been proposed that scalp recorded gamma-band oscillations in EEG-data reflect miniature saccades instead of cognitive or neuronal processes (Yuval-Greenberg et al., 2008). For a critical evaluation of this claim see Melloni et al. (2009). gamma-band oscillations across a wide frequency range during perceptual organization in schizophrenia.

In addition to analyses of spectral power, several studies have also examined phase synchrony between distributed neuronal populations while patients performed cognitive tasks (Slewa-Younan et al., 2004; Spencer et al., 2003; Uhlhaas et al., 2006). Overall, these studies conclude that patients with schizophrenia are characterised by reduced phase-locking of oscillations in the beta- and lower gamma-band range highlighting that in addition to abnormalities in local circuits, large-scale integration of neural activity is impaired. It is currently, unclear, however to what extent impairments in local circuits contribute to long-range synchronization impairments or whether these represent two independent phenomena.

Correlations between cognitive dysfunctions and alterations in neural synchrony are furthermore accompanied by relationships between the positive symptoms of schizophrenia and changes in the amplitude of beta- and gamma-band oscillations. Specifically, patients with auditory hallucinations are characterised by an increase in oscillatory activity in temporal regions relative to patients without hallucinations (Lee et al., 2006; Spencer et al., 2008), suggesting that the abnormal generation of internal experiences is related to increased beta- and gamma-band activity. These observations are also corroborated by the finding that auditory hallucinations in schizophrenia are associated with enhanced white matter connectivity in temporal regions as disclosed by recent DTI-studies (Hubl et al., 2004), indicating a possible link between pathological oscillatory activity and anatomical hyperconnectivity.

The observed deficits in neural synchrony in schizophrenia are consistent with impairments in neurotransmitter systems as well as altered anatomical parameters that are fundamental to establishing sychronised oscillations at high frequencies in cortical networks. One prominent candidate mechanism for the changes in neural synchrony are dysfunctions in GABAergic interneurons (see Lewis et al., 2005, for a review). For example, there is consistent evidence for reduced GABA ( $\gamma$-aminobutyric acid) synthesis in the parvalbumin-(PV) containing subpopulation of inhibitory GABA neurons in schizophrenia which are critically involved in the generation of cortical and hippocampal oscillatory activity (see 'The Generation of Neural Synchrony').

In addition, impairments in long-range synchronization in schizophrenia can be related to changes in white matter volume and organisation as long distance synchronization of oscillatory responses is mediated by reciprocal cortico-cortical connections (König et al., 1993; Löwel and Singer, 1992). This possibility is supported by in-vivo anatomical examinations with diffusion tensor imaging (DTI) that have revealed white matter anomalies throughout cortical and subcortical structures (for a review see Kubicki et al., 2007).

\section{AUTISM SPECTRUM DISORDERS (ASDS)}

ASDs constitute a heterogeneous neurodevelopmental syndrome that is characterized by a triad of impairments that affect social interaction, verbal and nonverbal communication, and the repertoire of interests and activities (APA, 2000). Similar to recent work in schizophrenia, theories that account for the pervasive cognitive dysfunctions associated with ASDs converge on the notion that the pathophysiological mechanisms in ASDs involve a deficit in 

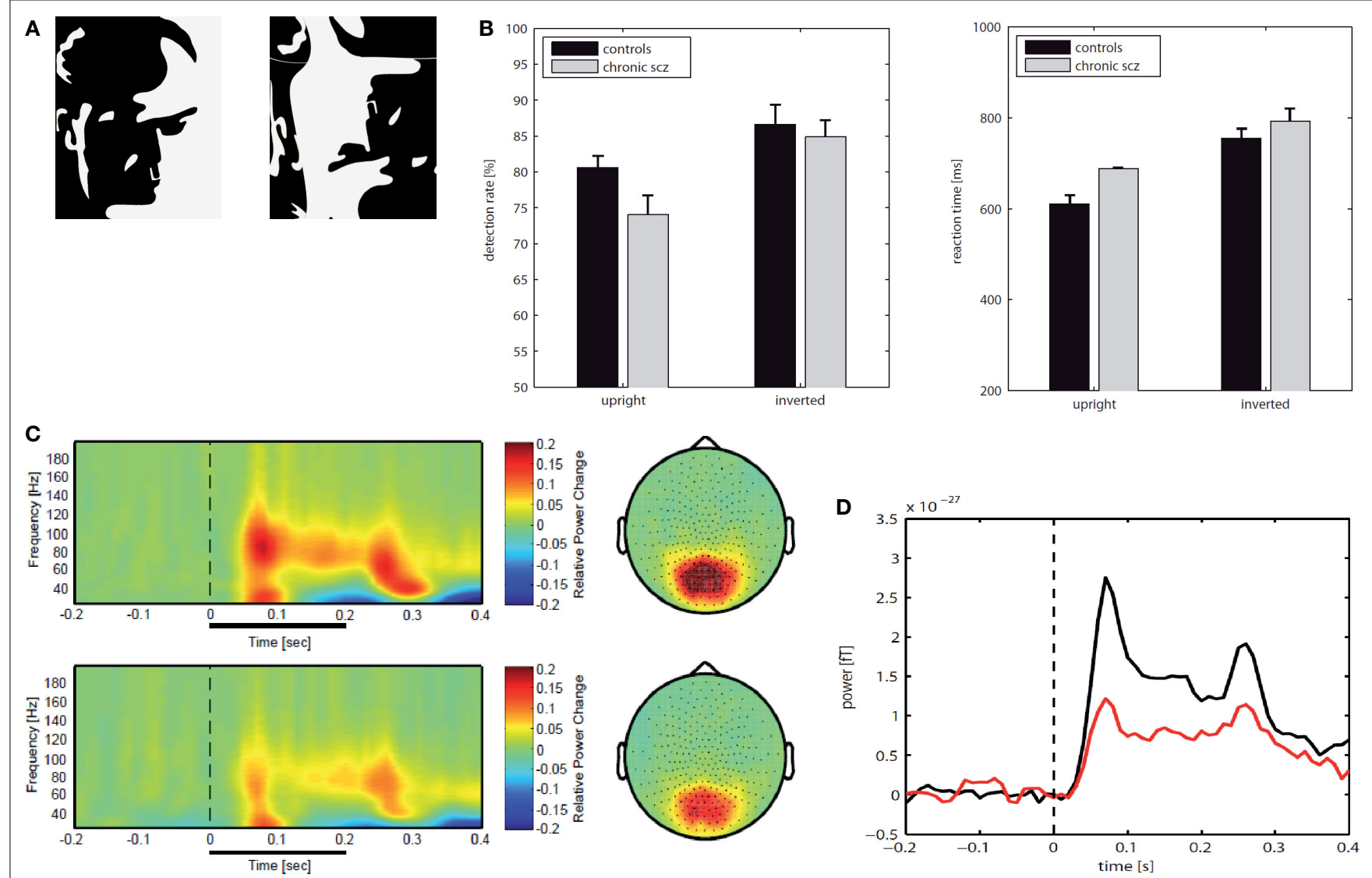

FIGURE 7 | (A) An upright image of a Mooney face and an inverted version of the same image. (B) Behavioral data for chronic patients with schizophrenia $(N=17)$ and matched healthy controls $(N=17)$; detection rates in percent (left) and reactions times (right). (C) Normalized evoked and induced spectral power for correct responses to upright
Mooney faces in the frequency range 25-200 Hz over parietal sensors; Top: normal controls, Bottom: patients with schizophrenia. (D) Time course for gamma-power $(60-120 \mathrm{~Hz})$ for parietal sensors during the perception of upright Mooney faces (red: schizophrenia patients; black: controls). the temporal coordination of distributed neural activity (Uhlhaas and Singer, 2007).

Parallels between the pathophysiology of schizophrenia and ASDs extent to cognitive impairments and certain symptoms. Thus, both disorders are characterised by abnormal perceptual integration during auditory and visual perception (Happe and Frith, 2006; Uhlhaas and Silverstein, 2005), for example, suggesting similar mechanisms that account for cognitive dysfunctions. Furthermore, clinical symptoms, such as the disorganization of thought and language as well as negative symptoms can be found in both disorders (Konstantareas and Hewitt, 2001), suggesting an overlap in the clinical phenotype.

Recent fMRI and EEG/MEG studies have supported the potential role of dysfunctional temporal coordination of neural responses in ASDs. Just et al. (2004) examined functional connectivity by measuring the covariance of BOLD signals during sentence comprehension in high-functioning individuals with autism. The study showed systematic differences between groups with respect to the distribution of brain activation and functional connectivity. Compared to controls, subjects with autism were characterized by a marked reduction in functional connectivity throughout the cortical language system that was most pronounced during comprehension of sentences. A number of additional fMRI studies have supported the concept of reduced functional connectivity in ASDs (Castelli et al., 2002; Koshino et al., 2005; Villalobos et al., 2005).

In analogy to the findings in schizophrenia patients, these data predict that autism should be associated with reduced neural synchrony. Similar to patients with schizophrenia, ASDs are characterised by impaired auditory steady-state (ASS) potentials to stimulation in the $40 \mathrm{~Hz}$ range. Wilson et al. (2007) examined entrainment to $40 \mathrm{~Hz}$ stimulation in MEG-data in healthy controls and children and adolescent participants with ASDs. Participants with ASDs showed a marked reduction in gamma-band power that was particularly pronounced in the left hemisphere. Impairments in gamma-band activity elicited by auditory stimulation were also observed in first-degree relatives of proband with ASDs (Rojas et al., 2008), suggesting that dysfunctions in gamma-band activity are a potential endophenotype.

In addition, there is also evidence for impairments in taskrelated oscillations in ASDs. Milne et al. (2009) investigated visuoperceptual integration in 20 individuals with ASD and 20 control subjects with EEG. Visual evoked potentials elicited by Gabor 
patches were compared in participants with and without ASD and a source-localisation algorithm was employed to identify the generators of oscillatory activity. In those components located near the striate or extrastriate cortex, variations in the spatial frequency of stimuli exerted a smaller effect on induced increases in alpha- and gamma-band power in participants with ASD, suggesting abnormal modulation and recruitment of alpha- and gamma-band oscillations during perception integration in ASDs.

Finally, resting state EEG-data suggest a relationship between neural synchrony and aberrant brain development in ASDs. Thus, an excess of gamma-band activity has been reported in children with ASDs (Orekhova et al., 2007). In contrast, in adult participants reduced long-range coherence in resting-state EEG has been observed (Murias et al., 2007), suggesting that during later developmental stages, there is faulty maturation of cortico-cortical connections that leads to a disconnection syndrome (Geschwind and Levitt, 2007). This evidence is in agreement with anatomical data that indicate that during early development, white matter increases significantly more in ASDs than in normal children (Herbert et al., 2003). Evidence for a transient hypertrophy of white matter has also been found in previous studies (Courchesne et al., 2001) and later complemented by results suggesting exaggerated pruning to subnormal levels consistent with evidence for anatomical hypoconnectivity (Just et al., 2004; McCaffery and Deutsch, 2005).

Anatomical abnormalities are accompanied by alterations in neurotransmitter systems that may lead to an imbalance between excitation and inhibition, and in turn to hyperexcitability and unstable cortical networks (Hussman, 2001; Rubenstein and Merzenich, 2003). This hypothesis is consistent with abnormalities in GABAergic and glutamatergic transmitter systems in ASDs (for a review see DiCicco-Bloom et al., 2006).

\section{NEURAL SYNCHRONY IN BRAIN DISORDERS}

In summary, the potential role of neural synchrony in schizophrenia and ASDs is supported by close correlations between alterations in neural synchrony and cognitive impairments as well as through dysfunctions in transmitter-systems and anatomical parameters that are critical for neural synchrony to occur in cortical networks. Accordingly, we believe that further investigations into the role of neural synchrony in schizophrenia and ASDs are likely to be crucial for establishing valid pathophysiological models of these disorders.

This perspective is based on the fact that efforts to identify specific cortical regions that can account for the diverse impairments in cognition and explain the heterogeneous symptoms have so far been fruitless. Instead, the more plausible assumption is that different syndromes are related to distinct but overlapping pathologies in the coordination of distributed neuronal activity patterns that are revealed by the systematic investigation of the temporal and spatial organisation of neural synchrony across different frequency bands. This is undoubtedly a challenging task but such a research program will ultimately lead to better diagnostic tools for early diagnosis and intervention.

\section{CONCLUDING REMARKS}

In this paper, we have attempted to give an overview on the history and current status of concepts about the role of oscillations and synchrony in neuronal networks. Initially discovered in the visual system and conceptualized as a possible mechanism for dynamically binding perceptual elements into coherent object representations, it is becoming more and more evident that this discovery has wider implications for the understanding of neural codes and integrated brain functions than originally assumed. However, we also wish to emphasize that many open questions remain that are fundamental to our understanding of this phenomenon and need to be addressed by future research.

\section{THE BINDING BY SYNCHRONY HYPOTHESIS}

A still much debated issue is the role of neural synchrony in perceptual grouping and feature binding. Evidence regarding the binding hypothesis is heterogeneous. Numerous studies support the initial interpretation while others report negative evidence. Most studies agree that synchronization in the retina or early visual cortex reflects well some of the Gestalt criteria for perceptual grouping such as continuity of contours, similarity in feature space (colinearity, similar orientation) and common fate. However, it is clear that synchronization at early stages of processing tends to be restricted to closely spaced (a few millimetres at most) groups of neurons and does not generalize to all neurons engaged in the encoding of an extended object (for citations see main text). Because multisite recordings in higher visual areas are rare, little is known as to whether cells in inferotemporal cortex participating in the distributed representation of an object exhibit signs of object specific synchronization. The one study that has specifically addressed that issue indicates that this might indeed be the case (Hirabayashi and Miyashita, 2005). When monkeys recognize that component features of a face (mouth, nose, eyes etc.) actually form a face rather than a scrambled collection of features, neurons responding to the features transiently synchronize their responses. Thus, when investigating relations between perceptual grouping and synchronization, it is important to make educated guesses as to which binding functions are expected to occur at which level of processing. We consider it as likely that binding functions are realized at many different scales, whereby early processing stages can support only very local grouping operations while global, object specific grouping takes place only in the late, non-retinotopically organized areas. It may be that some of the negative findings are the result of the probably inappropriate assumption that all binding should be achieved at once already at low processing levels. Another difficulty in the testing of the binding by synchrony hypothesis is the transitory nature of synchronization. Numerous studies indicate, especially when performed in awake behaving animals, that epochs of synchronous firing may be very short (in the range of tens of milliseconds). This is in perfect agreement with the evidence that scene segmentation, perceptual grouping and object recognition can be achieved in less than 200 ms which leaves only a few tens of milliseconds per processing stage to accomplish its operations. Short episodes of synchronous firing may, however, not be detectable with conventional cross correlation techniques, especially if discharge rates are low as is the case under natural viewing conditions. In this case other methods such as the unitary event analysis (Gruen et al., 2002a,b) or its refined version Neuroxidence (Pipa et al., 2008) need to be applied. These methods can detect individual events of coincident firing and distinguish them from spurious 
coincidences even if the discharge rates of the neurons are not stationary. If grouping operations are based on brief episodes of synchronous firing, they would have passed undetected because so far all experiments on feature binding have relied on conventional cross correlation analysis. Since these novel methods have been tested successfully on experimental and simulated data, they can now be applied to clarify the question whether synchronization serves to tag responses to groupable features as related and to thereby support perceptual grouping and scene segmentation.

In designing these future studies it will have to be considered that synchronization is state and attention dependent and that different binding functions are likely to be accomplished at different levels of the processing hierarchy. Thus, the challenge is to design tests that are appropriate for the investigated processing stage.

\section{FURTHER CHALLENGES}

Ideally, in all investigations of the functional role of synchronized oscillations, one would of course like to obtain causal evidence. This would require manipulations of oscillations and synchrony without interfering with other response variables. First attempts to achieve this with pharmacological and genetic manipulations of synchronizing mechanisms have been made but much more work is needed. So far, the large majority of studies have provided only correlative evidence for the involvement of neural synchrony in cognitive and executive functions. Although the same problem exists for the relation between changes in firing rates and behavioural functions, the possibility needs to be considered that changes in neural synchrony are in part an epiphenomenon of neuronal dynamics and are not exploited further. At present we consider this as highly unlikely because of the numerous findings from normal and abnormal brains that demonstrated close relationships between alterations in behaviour and cognition and associated modulations in the synchronization and amplitude of oscillatory activity in a wide range of frequencies. Also, there is abundant evidence from studies on synaptic plasticity and memory formation that emphasizes the importance of precise timing relations between the discharges of interconnected neurons for use dependent synaptic modifications (Markram et al., 1997). As timing relations are crucial in this context for the definition of relations, it follows that they must be of similar importance in signal processing because signatures of relatedness must be the same in signal processing and learning. As the timing relations relevant for determining the polarity of use dependent synaptic plasticity are defined on the scale of milliseconds, similarly precise temporal relations must be realized in signal processing in order to define the relatedness of neuronal responses.

Another important and unresolved question is related to the coexistence of oscillations in different frequency bands. We are only at the beginning of understanding their mutual interactions and spatial organization. Neuronal synchrony is found on different temporal and spatial scales. The spatial scale of synchrony can range from local synchronization of small numbers of neurons within a single cortical column to the synchronization of large populations that are distributed across different cortical regions and even across both hemispheres (Singer, 1999). The temporal scales of neuronal synchrony range from very precise sub-millisecond spike-spike synchronization to the synchronization of slow oscillatory activity as low as $<0.1 \mathrm{~Hz}$. Remarkably, synchronization is not confined to oscillations of the same frequency band but occurs across different frequencies as $n: m$ synchrony (Palva et al., 2005; Tognoli and Kelso, 2009). This allows for the concatenation of rhythms and for the establishment of partial correlations (Roopun et al., 2008). An attractive hypothesis is that this could serve as a mechanism to encode nested relations - an indispensable function for the neuronal representation of composite objects and movements. Yet, so far there is no evidence that the observed concatenation of rhythms is used for the encoding of graded, hierarchically organized relations.

Yet another conundrum of neural synchrony is that it can be stable and long lasting, e.g. in sleep or drowsiness, but also very dynamic and transient in awake and attentive brains, as it was reported for perceptual grouping and the control of focused attention (Fries et al., 2001a; Taylor et al., 2005). While stable synchrony can be explained by fixed coupling based on the connectivity of the network, the very transient switches between synchronized and unsynchronized dynamics are much more challenging properties regarding the underlying mechanism.

To explain the fast dynamical modulation of synchrony remains a theoretical challenge. The fast time scale of switches between synchronized and de-synchronized states observed in the data seems to be incompatible with the speed of changes in the functional topology or the coupling strength that can be achieved with conventional mechanisms of synaptic plasticity. The same holds for putative mechanisms that could cause changes in transmission delays. Thus, the most likely option for the modulation of synchrony is to change the dynamical states of the coupled neuronal populations, such as the balance between excitation and inhibition (Brunel, 2000; Destexhe et al., 1999). Such state changes can achieve fast and flexible modulations of effective coupling among populations of neurons or areas of the brain. They can be induced by rate changes of sub populations, by changes in the balance of excitation and inhibition, and also by changes of the level of noise (Melloni et al., 2007; Ullner et al., 2009). To distinguish between these mechanisms will require close cooperation between theoreticians and experimentalists for the formulation of predictions and their testing.

Thus, much further work is required for the exploration of the various functions of oscillations and neural synchrony. Since the discovery of stimulus and context dependent synchronization of gamma oscillations some 20 years ago, we keep being intrigued by the growing variety of cognitive and executive functions that seem to exploit this mechanism. As far as prognoses are possible in basic research, we predict that application of massively parallel multisite recordings techniques - electrical or optical - and evaluation of temporal relations among distributed neuronal responses will continue to provide us with data that we consider indispensable for a deeper understanding of integrated cerebral functions.

\section{ACKNOWLEDGMENTS}

This work was supported by the Max Planck Society, the Hertie Foundation, an EU grant Project "Global Approach to Brain Activity: From Cognition to Disease" (GABA) Contract No. 043309, a grant from Deutsche Forschungsgemeinschaft (NI 708/2-1), and by the German Federal Ministry of Education and Research (BMBF) within the "Bernstein Focus: Neurotechnology" through research grant 01GQ0840. 


\section{REFERENCES}

American Psychiatric, and Association (2000). Diagnostic and Statistical Manual of Mental Disorders, Fourth Edn, Text Revision. Washington, DC, American Psychiatric Association.

Arecchi,F. T., Boccaletti,S., and Ramazza, P. (1999). Pattern formation and competition in nonlinear optics. Phys. Rep. Rev. Sect. Phys. Lett. 318, 1-83.

Azouz, R., and Gray, C. M. (2003). Adaptive coincidence detection and dynamic gain control in visual cortical neurons in vivo. Neuron 37, 513-523.

Bartos, M., Vida, I., and Jonas, P. (2007). Synaptic mechanisms of synchronized gamma oscillations in inhibitory interneuron networks. Nat. Rev. Neurosci. 8, 45-56.

Betsch, B. Y., Einhäuser, W., Körding, K. P., and König, P. (2004). The world from a cat's perspective - statistics of natural videos. Biol. Cybern. 90, 41-50.

Biederlack, J., Castelo-Branco, M., $\mathrm{Ne}$ u e n s chwand e r, S. Wheeler, D. W., Singer, W., and Nikolić, D. (2006). Brightness induction: rate enhancement and neuronal synchronization as complementary codes. Neuron 52, 1073-1083.

Bleuler, E. (1950). Dementia Praecox or the Group of Schizophrenias. New York, International University Press. (Original work published in 1911).

Brecht, M., Singer, W., and Engel, A. K. (1998). Correlation analysis of corticotectal interactions in the cat visual system. J. Neurophysiol. 79, 2394-2407.

Brunel, N. (2000). Dynamics of sparsely connected networks of excitatory and inhibitory spiking neurons. J. Comput. Neurosci. 8, 183-208.

Buia, C., and Tsienga, P. H. (2006). Attentional modulation of firing rate and synchrony in a model cortical network. J. Comput. Neurosci. 20, 247-264.

Buzsaki, G. (2002). Theta oscillations in the hippocampus. Neuron 33, 325-340.

Buzsaki, G. (2007). The structure of consciousness. Nature 446, 267.

Buzsaki, G., and Draguhn, A. (2004). Neuronal oscillations in cortical networks. Science 304, 1926-1929.

Cardin, J. A., Carlén, M., Meletis, K., Knoblich, U., Zhang, F., Deisseroth, K., Tsai, L. H., and Moore, C. I. (2009). Driving fast-spiking cells induces gamma rhythm and controls sensory responses. Nature 459, 663-667.

Carrasco, M., Ling, S., and Read, S. (2004). Adaptive coincidence detection and dynamic gain control in visual cortical neurons in vivo. Nat. Neurosci. 7, 308-313.

Castelli, F., Frith, C., Happe, F., and Frith, U. (2002). Autism, Asperger syndrome and brain mechanisms for the attribution of mental states to animated shapes. Brain 125, 1839-1849.

Chalmers, D. J. (2000). What is a neural correlate of consciousness? In Neural Correlates of Consciousness: Empirical and Conceptual Questions, T. Metzinger, ed. (Cambridge, MA, MIT Press), pp. 17-40.

Cosmelli, D., David, O., Lachaux, J. P., Martinerie, J., Garnero, L., Renault, B., and Varela, F. (2004). Waves of consciousness: ongoing cortical patterns during binocular rivalry. Neuroimage 23, 128-140.

Courchesne, E., Karns, C.M., Davis, H. R. Ziccardi, R., Carper, R. A., Tigue, Z. D., Chisum, H. J., Moses, P., Pierce, K., Lord, C., Lincoln, A. J., Pizzo, S., Schreibman, L., Haas, R. H., A kshoomoff, N. A., and Courchesne, R. Y. (2001). Unusual brain growth patterns in early life in patients with autistic disorder: an MRI study. Neurology 57, 245-254.

Dehaene, S., Changeux, J. P., Naccache, L., Sackur, J., and Sergent, C. (2006). Conscious, preconscious, and subliminal processing: a testable taxonomy. Trends Cogn. Sci. 10, 204-211.

Dehaene, S., Naccache, L., Cohen, L., Bihan, D. L., Mangin, J. F., Poline, J. B., and Riviere, D. (2001). Cerebral mechanisms of word masking and unconscious repetition priming. Nat Neurosci. 4, 752-758.

Dehaene, S., Naccache, L., Le Clec, H. G., Koechlin, E., Mueller, M., DehaeneLambertz, G., van de Moortele, P. F., and Le Bihan, D. (1998). Imaging unconscious semantic priming. Nature 395, 597-600.

Desimone, R., and Duncan, J. (1995). Neural mechanisms of selective visual attention. Annu. Rev. Neurosci. 18, 193-222.

Destexhe,A.,Contreras,D., and Steriade, M. (1999). Cortically-induced coherence of a thalamic-generated oscillation. Neuroscience 92, 427-443.

DiCicco-Bloom, E., Lord, C. Zwaigenbaum, L., Courchesne, E., Dager, S. R., Schmitz, C., Schultz, R. T. Crawley, J., and Young, L. J. (2006). The developmental neurobiology of autism spectrum disorder. J. Neurosci. 26, 6897-6906.

Doesburg, S.M., Kitajo, K., andWard, L. M. (2005). Increased gamma-band synchrony precedes switching of conscious perceptual objects in binocular rivalry. Neuroreport 16, 1139-1142.

Doesburg, S. M., Roggeveen, A. B., Kitajo, K., and Ward, L. M. (2008). Large-scale gamma-band phase synchronization and selective attention. Cereb. Cortex 18, 386-396.

Dong, Y., Mihalas, S., Qiu, F., von der Heydt, R., and Niebur, E. (2008).
Synchrony and the binding problem in macaque visual cortex. J. Vis. 8, 1-16.

Eckhorn, R., Frien, A., Bauer, R. Woelbern, T., and Kehr, H. (1993). High frequency $(60-90 \mathrm{~Hz})$ oscillations in primary visual cortex of awake monkey. Neuroreport 4, 243-246.

Engel,A. K., Fries, P., and Singer, W. (2001). Dynamic predictions: oscillations and synchrony in top-down processing Nat. Rev. Neurosci. 2, 704-716.

Engel, A. K., König, P., Kreiter, A. K., and Singer,W.(1991).Stimulus-dependent neuronal oscillations in cat visual cortex: inter-columnar interactions as determined by cross-correlation analysis. Eur. J. Neurosci. 2, 588-606.

Engel, A. K., and Singer, W. (2001) Temporal binding and the neural correlates of sensory awareness. Trend Cogn. Sci. 5, 16-25.

Fan,J.,Byrne,J., Worden, M.S., Guise, K. G. McCandliss, B. D., Fossella, J., and Posner, M. I. (2007). The relation of brain oscillations to attentional networks. J. Neurosci. 27, 6197-6206.

Ferrarelli, F., Massimini, M., Peterson, M. J. Riedner, B.A., Lazar, M., Murphy, M. J., Huber,R.,Rosanova,M.,Alexander, A. L. Kalin, N., and Tononi, G. (2008) Reduced evoked gamma oscillations in the frontal cortex in schizophrenia patients: a TMS/EEG study. Am. J. Psychiatry. 165, 996-1005.

Fischer, I., Vicente, R., Buldu, J. M., Peil, M., Mirasso, C. R., Torrent, M. C., and Garcia-Ojalvo, J. (2006). Zerolag long-range synchronization via dynamical relaying. Phys. Rev. Lett. 97, 123902.

Frien, A., and Eckhorn, R. (2000). Functional coupling shows stronger stimulus dependency for fast oscillations than for low-frequency components in striate cortex of awake monkey. Eur. J. Neurosci. 12, 1466-1478.

Fries, P. (2005). A mechanism for cognitive dynamics: neuronal communication through neuronal coherence. Trends Cogn. Sci. 9, 474-480.

Fries, P., Reynolds, J. H., Rorie, A. E., and Desimone, R. (2001a). Modulation of oscillatory neuronal synchronization by selective visual attention. Science 291, 1560-1563.

Fries, P., Neuenschwander, S., Engel,A. K., Goebel, R., and Singer, W. (2001b). Rapid feature selective neuronal synchronization through correlated latency shifting. Nature Neurosci. 4 , 194-200.

Fries, P., Nikolić, D., and Singer, W. (2007) The gamma cycle. Trends Neurosci.30, 309-316.

Fries, P., Roelfsema, P. R., Engel, A. K., König, P., and Singer, W. (1997). Synchronization of oscillatory responses in visual cortex correlates with perception in interocular rivalry. Proc. Natl. Acad. Sci. U.S.A. 94, 12699-12704.

Fries, P., Schröder, J. H., Roelfsema, P. R., Singer, W., and Engel, A. K. (2002). Oscillatory neuronal synchronization in primary visual cortex as a correlate of stimulus selection. J. Neurosci. 22, 3739-3754.

Fries, P., Womelsdorf, T., Oostenveld, R., and Desimone, R. (2008). The effects of visual stimulation and selective visual attention on rhythmic neuronal synchronization in macaque area V4. J. Neurosci. 28, 4823-4835.

Friston, K. J. (1999). The disconnection hypothesis. Schizophr. Res. 30, 115-125.

Gail, A., Brinksmeyer, H. J., and Eckhorn, R. (2000). Contour decouples gamma activity across texture representation in monkey striate cortex. Cereb. Cortex 10, 840-850.

Gallinat, J., Winterer, G., Herrmann, C. S., and Senkowski, D. (2004). Reduced oscillatory gamma-band responses in unmedicated schizophrenic patients indicate impaired frontal network processing. Clin. Neurophysiol. 115, 1863-1874.

Geschwind, D. H., and Levitt, P. (2007). Autism spectrum disorders: developmental disconnection syndromes. Curr. Opin. Neurobiol. 17, 103-111.

Ghose, G. M., and Maunsell, J. (1999). Specialized representations in visual cortex: a role for binding? Neuron 24, 79-85, 111-125.

Gray, C. M., Koenig, P., Engel, A. K., and Singer,W. (1989). Oscillatory responses in cat visual cortex exhibit inter-columnar synchronization which reflects global stimulus properties. Nature 23, 334-337.

Gray, C.M., and Mc Cormick, D.A. (1996). Chattering cells: superficial pyramidal neurons contributing to the generation of synchronous oscillations in the visual cortex. Science 274, 109-113.

Gray, C. M., and Viana Di Prisco, G. (1997). Stimulus-dependent neuronal oscillations and local synchronization in striate cortex of the alert cat. J. Neurosci. 17, 3239-3253.

Grinstein, G., and Linsker, R. (2005). Synchronous neural activity in scale free network models versus random network models. Proc. Natl. Acad. Sci. U.S.A. 102, 9948-9953.

Gross, J., Schmitz, F., Schnitzler, I., Kessler, K., Shapiro, K., Hommel, B., and Schnitzler, A. (2004). Modulation of long-range neural synchrony reflects temporal limitations of visual attention in humans. Proc. Natl. Acad. Sci. U.S.A. 101, 13050-13055.

Gruen, S., Diesmann, M., and Aertsen, A. M. H. J. (2002a). Unitary 
events in multiple single-neuron spiking activity: I. Detection and significance. Neural Comput. 14, 43-80.

Gruen, S., Diesmann, M., and Aertsen, A. M. H. J. (2002b). Unitary events in multiple single-neuron spiking activity: II. Nonstationary data. Neural Comput. 14, 81-119.

Happé, F., and Frith, U. (2006). The weak coherence account: detail-focused cognitive style in autism spectrum disorders. J. Autism. Dev. Dis. 36, 5-25.

Havenith, M. N., Yu, S., Chen, N., Biederlack, J., Singer, W., and Nikolic, D. (2007). Millisecond delays in neuronal spiking activity carry information as accurately as firing rates. Soc. Neurosci. Abstr. 615.7 2007: Society for Neuroscience 37th Annual Meeting, San Diego, USA.

Havenith, M. N., Zemmar, A., Yu, S., Baudrexel, S. M., Singer, W., and Nikolić, D. (2009). Measuring submillisecond delays in spiking activity with millisecond time-bins. Neurosci. Lett. 450, 296-300.

Herbert, M. R., Ziegler, D. A., Deutsch, C. K., O'Brien, L. M., Lange, N., Bakardjiev, A., Hodgson, J., Adrien, K. T., Steele, S., Makris, N., Kennedy, D., Harris, G. J., and Caviness, V. S. (2003). Dissociations of cerebral cortex, subcortical and cerebral white matter volumes in autistic boys. Brain 126, 1182-1192.

Herculano-Houzel, S., Munk, M. H., Neuenschwander, S., and Singer, W. (1999). Precisely synchronized oscillatory firing patterns require electroencephalographic activation. J. Neurosci. 19, 3992-4010.

Herrmann, C. S., Munk, M. H. J., and Engel, A. K. (2004). Cognitive functions of gamma-band activity: memory match and utilization. Trends Cogn. Sci. 8, 347-355.

Hirabayashi, T., and Miyashita, Y. (2005). Dynamically modulated spike correlation in monkey inferior temporal cortex depending on the feature configuration within a whole object. J. Neurosci. 25, 10299-10307.

Huang, D., and Pipa, G. (2007). Achieving synchronization of networks by an auxiliary hub. Europhys. Lett. 77, 50010.

Hubl, D., Koenig, T., Strik, W., Federspiel, A., Kreis, R., Boesch, C., Maier, S. E., Schroth, G., Lovblad, K., and Dierks, T. (2004). Pathways that make voices: white matter changes in auditory hallucinations. Arch. Gen. Psychiatry 61, 658-668.

Hussman, J. P. (2001). Suppressed GABAergic inhibition as a common factor in suspected etiologies of autism. J. Autism Dev. Disord. 31, 247-248.
Jensen, O., Kaiser, J., and Lachaux, J. P. (2007). Human gamma-frequency oscillations associated with attention and memory. Trends Neurosci. 30, 317-324.

Jensen, O., and Tesche, C. D. (2002). Frontal theta activity in humans increases with memory load in a working memory task. Eur. J. Neurosci. 15, 1395-1399.

Just, M. A., Cherkassky, V. L., Keller, T. A., and Minshew, N. J. (2004). Cortical activation and synchronization during sentence comprehension in high-functioning autism: evidence of underconnectivity. Brain 127, 1811-1821.

Kanwisher, N. (2001). Neural events and perceptual awareness. Cognition 79, 89-113.

König, P., Engel, A. K., Löwel, S., and Singer, W. (1993). Squint affects synchronization of oscillatory responses in cat visual cortex. Eur. J. Neurosci. 5, 501-508.

König, P., Engel, A. K., Roelfsema, P. R., and Singer, W. (1995). How precise is neuronal synchronization? Neural Comput. 7, 469-485.

Konstantareas, M. M., and Hewitt, T. (2001). Autistic disorder and schizophrenia: diagnostic overlaps. J. Autism Dev. Disord. 31, 19-28.

Kopell, N., and Ermentrout, B. (2004). Chemical and electrical synapses perform complementary roles in the synchronization of interneuronal networks. Proc. Natl. Acad. Sci. U.S.A. 101, 15482-15487.

Kopell, N., Ermentrout, G. B., Whittington, M. A., and Traub, R. D. (2000). Gamma rhythms and beta rhythms have different synchronization properties. Proc. Natl. Acad. Sci. U.S.A. 97, 1867-1872.

Koshino, H., Carpenter, P. A., Minshew, N. J., Cherkassky, V. L., Keller, T. A., and Just, M. A. (2005). Functional connectivity in an fMRI working memory task in highfunctioning autism. NeuroImage 24 810-821.

Kreiter, A. K., and Singer, W. (1992). Oscillatory neuronal responses in the visual cortex of the awake macaque monkey. Eur. J. Neurosci. 4, 369-375.

Kreiter, A. K., and Singer, W. (1996). Stimulus-dependent synchronization of neuronal responses in the visual cortex of the awake macaque monkey. J. Neurosci. 16, 2381-2396.

Krishnan, G. P., Vohs, J. L., Hetrick, W. P., Carroll, C. A., Shekhar, A., Bockbrader, M. A., and O'Donnell, B. F. (2005). Steady state visual evoked potential abnormalities in schizophrenia. Clin. Neurophysiol. $116,614-624$.
Kubicki, M., McCarley, R., Westin, C. F., Park, H. J., Maier, S., Kikinis, R. Jolesz, F.A., and Shenton, M.E. (2007) A review of diffusion tensor imaging studies in schizophrenia. J. Psychiatr. Res. 41, 15-30.

Kwon, J. S., O'Donnell, B. F., Wallenstein, G. V., Greene, R. W., Hirayasu, Y., Nestor, P. G., Hasselmo, M. E., Potts, G. F., Shenton, M. E., and McCarley, R. W. (1999). Gamma frequency-range abnormalities to auditory stimulation in schizophrenia. Arch. Gen. Psychiatry 56, 1001-1005.

Lakatos, P., Chen, C. M., O'Connell, M.N., Mills, A., and Schroeder, C. E. (2007). Neuronal oscillations and multisensory interaction in primary auditory cortex. Neuron 53, 279-292.

Lakatos, P., Karmos, G., Mehta, A. D., Ulbert, I., and Schroeder, C. E. (2008). Entrainment of neuronal oscillations as a mechanism of attentional selection. Science 320, 110-113.

Lamme, V. A., and Spekreijse, H. (1998). Neuronal synchrony does not represent texture segregation. Nature 396, 362-366.

Lee, S. H., Wynn, J. K., Green, M. F., Kim, H., Lee, K. J., Nam, M., Park, J.K., and Chung, Y. C. (2006). Quantitative EEG and low resolution electromagnetic tomography (LORETA) imaging of patients with persistent auditory hallucinations. Schizophr. Res. 83, 111-119.

Lewis, D. A., Hashimoto, T., and Volk, D. W. (2005). Cortical inhibitory neurons and schizophrenia. Nat. Rev. Neurosci. 6, 312-324.

Lima, B., Chen, N., Singer, W., and Neuenschwander,S. (2006). Strength of stimulus-induced gamma oscillations in monkey V1 depend on attention and expectancy. Soc. Neurosci. Abstr. 734.5 Society for Neuroscience 36th Annual Meeting, Washington, DC, USA.

Lima, B., Singer, W., and Neuenschwander, S. (2007). Distributed gamma oscillations induced by expectancy in monkey V1 and V4. Soc. Neurosci. Abstr. 338.5 Society for Neuroscience 37th Annual Meeting, Washington, DC, USA

Llinas, R., and Pare, D. (1998). Coherent oscillations in specific and nonspecific thalamo-cortical networks and their role in cognition. In Thalamus, E. G Jones, ed. (Amsterdam, Elsevier), pp. 501-516.

Löwel, S., and Singer, W. (1992). Selection of intrinsic horizontal connections in the visual cortex by correlated neuronal activity. Science 255, 209-212.

Lutz, A., Lachaux, J. P., Martinerie, J., and Varela,F.J. (2002). Guiding the study of brain dynamics by using first-person data: synchrony patterns correlate with ongoing conscious states during a simple visual task. Proc. Natl. Acad. Sci. U.S.A. 99, 1586-1591.

Maldonado, P. E., Friedman-Hill, S., and Gray, C. M. (2000). Dynamics of striate cortical activity in the alert macaque: II. Fast time scale synchronization. Cereb. Cortex 10, 1117-1131.

Markram, H., Lübke, J., Frotscher, M., and Sakmann, B. (1997). Regulation of synaptic efficacy by coincidence of postsynaptic APs and EPSPs. Science 275, 213-215.

McCaffery, P., and Deutsch, C. K. (2005). Macrocephaly and the control of brain growth in autistic disorders. Prog. Neurobiol. 77, 38-56.

Melloni,L.,Molina, C.,Pena, M., Torres, D., Singer, W., and Rodriguez, E. (2007). Synchronization of neural activity across cortical areas correlates with conscious perception. J. Neurosci. 27, 2858-2865.

Melloni, L., and Rodriguez, E. (2007). Non-perceived stimuli elicit local but not large-scale neural synchrony. Perception 36, (ECVP Abstract Supplement).

Melloni, L., Schwiedrzik, C. M., Rodriguez, E., and Singer, W. (2009). (Micro)Saccades, corollary activity and cortical oscillations. Trends Cogn. Sci. 3, 239-245.

Milne, E., Scope, A., Pascalis, O., Buckley, D., and Makeig, S. (2009). Independent component analysis reveals atypical electroencephalographic activity during visual perception in individuals with autism. Biol. Psychiatry 65, 22-30.

Milo, R., Shen-Orr, S., Itzkovitz, S., Kashtan, N., Chklovskii, D., and Alon, U. (2002). Network motifs: simple building blocks of complex networks. Science 298, 824-827.

Munk, M. H., Roelfsema, P. R., König, P., Engel, A. K., and Singer, W. (1996). Role of reticular activation in the modulation of intracortical synchronization. Science 272, 271-274.

Murias, M., Webb, S. J., Greenson, J., and Dawson, G. (2007). Resting state cortical connectivity reflected in EEG coherence in individuals with autism. Biol. Psychiatry 62, 270-273.

Neuenschwander, S., Engel, A. K., König, P., Singer, W., and Varela, F. J. (1996). Synchronization of neuronal responses in the optic tectum of awake pigeons. Vis. Neurosci. 13, 575-584.

Neuenschwander, S., Lima, B., and Singer, W. (2008). Stimulus and task-related gamma oscillations in monkey V1 induced by local and global contours. Soc. Neurosci. Abstr. 2008-S-112007-SfN. Society for 
Neuroscience 38th Annual Meeting, Washington, DC, USA.

Neuenschwander, S., and Singer, W. (1996). Long-range synchronization of oscillatory light responses in the cat retina and lateral geniculate nucleus. Nature 379, 728-732.

Nikolić, D. (2007). Non-parametric detection of temporal order across pairwise measurements of time delays. J. Comput. Neurosci. 22, 5-19.

Nikolić, D., Häusler, S., Singer, W., and Maass, W. (2007). Temporal dynamics of information content carried by neurons in the primary visual cortex. Adv. Neural Inf. Process. Syst. 19.

Nikolić, D.,Schneider, G.,Havenith, M. N., and Singer, W. (2004). Short time delays in neuronal activity depend on stimulus properties. Soc. Neurosci. Abstr. 490.8. 2004: Society for Neuroscience 34th Annual Meeting, San Diego, USA.

O'Keefe, J., and Recce, M. L. (1993). Phase relationship between hippocampal place units and the EEG theta rhythm. Hippocampus 3, 317-330.

Orekhova, E. V., Stroganova, T. A., Nygren, G., Tsetlin, M. M., Posikera, I. N., Gillberg, C., and Elam, M. (2007). Excess of high frequency electroencephalogram oscillations in boys with autism. Biol. Psychiatry 62, 1022-1029.

Palanca, B. J., and DeAngelis, G. C. (2005). Does neuronal synchrony underlie visual feature grouping? Neuron 46, 333-346.

Palva, J. M., Palva, S., and Kaila, K. (2005). Phase synchrony among neuronal oscillations in the human cortex. J. Neurosci. 25, 3962-3972.

Palva, S., and Palva, J. M. (2007). New vistas for alpha-frequency band oscillations. Trends Neurosci. 30, 150-158.

Pesaran, B., Pezaris, J. S., Sahani, M., Mitra, P. P., and Andersen, R. A. (2002). Temporal structure in neuronal activity during working memory in macaque parietal cortex. Nat. Neurosci. 5, 805-811.

Phillips, W. A., and Silverstein, S. M. (2003). Convergence of biological and psychological perspectives on cognitive coordination in schizophrenia. Behav. Brain Sci. 26, 65-138.

Pikovsky,A., Rosenblum, M., and Kurths, J. (2001). Synchronization. Cambridge, Cambridge University Press.

Pipa, G., Riehle, A., and Grün, S. (2007). Validation of task-related excess of spike coincidences based on NeuroXidence. Neurocomputing 70, 2064-2068.

Pipa, G., Wheeler, D. W., Singer, W., and Nikolić, D. (2008). NeuroXidence: reliable and efficient analysis of an excess or deficiency of joint-spike events. J. Comput. Neurosci. 25, 64-88.
Posner, M. I. (1980). Orienting of attention. Q. J. Exp. Psychol. 32, 3-25.

Reynolds, J. H., and Chelazzi, L. (2004). Attentional modulation of visual processing. Annu. Rev. Neurosci. 27, 611-647.

Reynolds, J. H., and Desimone, R. (2000). The role of neural mechanisms of attention in solving the binding problem. Neuron 24, 19-29, 111-125.

Ritz, R., and Sejnowski, T. J. (1997). Synchronous oscillatory activity in sensory systems: new vistas on mechanisms. Curr. Opin. Neurobiol. 7, 536-546.

Roberts, M., Delicato, L. S., Herrero, J., Gieselmann, M. A., and Thiele, A. (2007). Attention alters spatial integration in macaque $\mathrm{V} 1$ in an eccentricitydependent manner. Nat. Neurosci. 10, 1483-1491.

Rodriguez, E., George, N., Lachaux, J. P., Martinerie, J., Renault, B., and Varela, F. J. (1999). Perception's shadow: long-distance synchronization of human brain activity. Nature 397, 430-433.

Rodriguez, R., Kallenbach, U., Singer, W., and Munk, M. H. J. (2004). Short- and long-term effects of cholinergic modulation on gamma oscillations and response synchronization in the visual cortex. J. Neurosci. 24, 10369-10378.

Roelfsema, P. R. (2006). Cortical algorithms for perceptual grouping. Annu. Rev. Neurosci. 29, 203-227.

Roelfsema, P. R., Engel, A. K., König, P., and Singer, W. (1997). Visuomotor integration is associated with zero time-lag synchronization among cortical areas. Nature 385, 157-161.

Roelfsema, P. R., Lamme, V. A., and Spekreijse, H. (2004). Synchrony and covariation of firing rates in the primary visual cortex during contour grouping. Nat. Neurosci. 7, 982-991.

Rojas, D. C., Maharajh, K., Teale, P., and Rogers, S. J. (2008). Reduced neural synchronization of gamma-band MEG oscillations in first-degree relatives of children with autism. BMC Psychiatry 8, 66.

Roopun, A. K., Kramer, M. A., Carracedo, L. M., Kaiser, M., Davies, C. H., Traub, R. D., Kopell, N. J., and Whittington, M. A. (2008). Period concatenation underlies interactions between gamma and beta rhythms in neocortex. Front. Cell. Neurosci. 2, 1.

Roy, A., Steinmetz, P. N., Hsiao, S. S., Johnson, K. O., and Niebur, E. (2007). Synchrony: a neural correlate of somatosensory attention. J. Neurophysiol. 98, 1645-1661.

Rubenstein, J. L., and Merzenich, M. M. (2003). Model of autism: increased ratio of excitation/inhibition in key neural systems. Genes Brain Behav. 2, 255-267.

Salinas, E., and Sejnowski, T. J. (2000) Impact of correlated synaptic input on output firing rate and variability in simple neuronal models. J. Neurosci. 20, 6193-6209.

Salinas, E., and Sejnowski, T. J. (2001) Correlated neuronal activity and the flow of neural information. Nat. Rev. Neurosci. 2, 539-550.

Schack, B., Klimesch, W., and Sauseng, P. (2005). Phase synchronization between theta and upper alpha oscillations in a working memory task. Int J. Psychophysiol. 57, 105-114.

Schneider, G., Havenith, M. N., and Nikolić, D. (2006). Spatio-temporal structure in large neuronal networks detected from cross-correlation. Neural Comput. 18, 2387-2413.

Schneider, G., and Nikolić, D. (2006) Detection and assessment of near-zero delays in neuronal spiking activity. J. Neurosci. Meth. 152, 97-106.

Schoffelen, J. M., Oostenveld, R., and Fries, P. (2005). Neuronal coherence as a mechanism of effective corticospinal interaction. Science 308, 111-113.

Schuster, H. G., and Wagner, P. (1989). Mutual entrainment of two limit cycle oscillators with time delayed coupling. Prog. Theor. Phys. 81, 939-945.

Sehatpour,P., Molholm, S., Schwartz, T. H., Mahoney, J. R., Mehta,A. D., Javitt, D. C., Stanton, P. K., and Foxe, J. J. (2008). A human intracranial study of long-range oscillatory coherence across a frontal-occipitalhippocampal brain network during visual object processing. Proc. Natl. Acad. Sci. U.S.A. 105, 4399-4404.

Sejnowski, T. J., and Paulsen, O. (2006). Network oscillations: emerging computational principles. J. Neurosci. 26 1673-1676.

Sergent, C., Baillet, S., and Dehaene, S (2005). Timing of the brain events underlying access to consciousness during the attentional blink. Nat. Neurosci. 8, 1391-1400.

Shadlen, M.N., and Movshon, J.A. (1999) Synchrony unbound: a critical evaluation of the temporal binding hypothesis. Neuron 24, 67-77, 111-125.

Sheinberg, D. L., and Logothetis, N. K. (2001). Noticing familiar objects in real world scenes: the role of temporal cortical neurons in natural vision. J. Neurosci. 21, 1340-1350.

Sherman, S. M., and Guillery, R.W.(2002) The role of the thalamus in the flow of information to the cortex. Philos. Trans. R. Soc. Lond., B, Biol. Sci. 357, 1695-1708.

Singer, W. (1999). Neuronal synchrony: a versatile code for the definition of relations? Neuron 24, 49-65, 111-125.
Singer,W. (2002). Phenomenal awareness and consciousness from a neurobiological perspective. In Neural Correlates of Consciousness, T. Metzinger, ed. (Cambridge, MA, MIT Press), pp. 121-137.

Singer, W. (2004). Time as coding space in the cerebral cortex. In Functional Neuroimaging of Visual Cognition. Attention and Performance XX., N. Kanwisher and J.Duncan. eds (Oxford, Oxford University Press), pp. 99-123.

Slewa-Younan, S., Gordon, E., Harris, A. W., Haig, A. R., Brown, K. J., Flor-Henry, P., and Williams, L. M. (2004). Sex differences in functional connectivity in first-episode and chronic schizophrenia patients. Am. J. Psychiatry 161, 1595-1602.

Sohal, V. S., Zhang, F., Yizhar, O., and Deisseroth, K. (2009). Parvalbumin neurons and gamma rhythms enhance cortical circuit performance. Nature 459, 698-702.

Spencer, K. M., Nestor, P. G., Niznikiewicz, M. A., Salisbury, D. F., Shenton, M. E., and McCarley, R. W. (2003). Abnormal neural synchrony in schizophrenia. J. Neurosci. 23, 7407-7411.

Spencer, K.M., Nestor,P.G., Perlmutter, R. Niznikiewicz, M. A., Klump, M. C., Frumin, M., Shenton, M. E., and McCarley, R. W. (2004). Neural synchrony indexes disordered perception and cognition in schizophrenia. Proc. Natl. Acad. Sci. U.S.A. 101, 17288-17293.

Spencer, K. M., Salisbury, D. F., Shenton, M. E., and McCarley, R. W. (2008). Gamma-band auditory steady-state responses are impaired in first episode psychosis. Biol. Psychiatry 64, 369-375.

Spitzer, H., Desimone, R., and Moran, J. (1988). Increased attention enhances both behavioral and neuronal performance. Science 240, 338-340.

Sporns, O., and Kotter, R. (2004). Motifs in brain networks. PLOS Biol. 2, 1910-1918.

Srinivasan, R., Russell,D.P.,Edelman, G. M., and Tononi, G. (1999). Increased synchronization of neuromagnetic responses during conscious perception. J. Neurosci. 19, 5435-5448.

Steinmetz, P. N., Roy, A., Fitzgerald, P. J., Hsiao, S.S., Johnson, K.O., and Niebur, E. (2000). Attention modulates synchronized neuronal firing in primate somatosensory cortex. Nature 404, 187-190.

Steriade, M., Mccormick, D. A., and Sejnowski, T.J.(1993). Thalamocortical oscillations in the sleeping and aroused brain. Science 262, 679-685.

Tallon-Baudry, C. (2009). The roles of gamma-band oscillatory synchrony in human visual cognition. Front. Biosci. 14, 321-332. 
Tallon-Baudry, C., Bertrand, O., Delpuech, C., and Permier, J. (1997). Oscillatory gamma-band $(30-70 \mathrm{~Hz})$ activity induced by a visual search task in humans. J. Neurosci. 17, 722-734.

Taylor, K., Mandon, S., Freiwald, W. A., and Kreiter, A. K. (2005). Coherent oscillatory activity in monkey area V4 predicts successful allocation of attention. Cereb. Cortex 15 , 1424-1437.

Thiele, A., and Stoner, G. (2003). Neuronal synchrony does not correlate with motion coherence in cortical area MT. Nature 421, 366-370.

Thompson, E., and Varela, F. J. (2001). Radical embodiment: neural dynamics and consciousness. Trends $\operatorname{Cog} n$. Sci. 5, 418-425.

Tiesinga, P.H., and Sejnowski, T. J. (2004). Rapid temporal modulation of synchrony by competition in cortical interneuron networks. Neural Comput. $16,251-275$.

Tillmann, C., Wibral, M., Leweke, M., Kohler, A., Singer, W., Koethe, D., Kranaster, L., Maurer, K., and Uhlhaas, P. J. (2008). High-frequency gamma-band oscillations during perceptual organisation in chronic and first-episode schizophrenia patients. Soc. Neurosci. Abstr. 54, 2. Society for Neuroscience 38th Annual Meeting, Washington, D.C., USA.

Tognoli, E., and Kelso, J. A. (2009). Brain coordination dynamics: true and false faces of phase synchrony and metastability. Prog. Neurobiol. 87, 31-40.

Tononi, G., Srinivasan, R., Russell, D. P., and Edelman, G. M. (1998). Investigating neural correlates of conscious perception by frequency-tagged neuromagnetic responses. Proc. Natl. Acad. Sci. U.S.A. 95, 3198-3203.

Traub, R. D., Bibbig, A., LeBeau, F. E., Buhl, E. H., and Whittington, M. A. (2004). Cellular mechanisms of neuronal population oscillations in the hippocampus in vitro. Annu. Rev. Neurosci. 27, 247-278.

Traub, R. D., Whittington, M. A., Stanford, I. M., and Jefferys, J. G. R. (1996). A mechanism for generation of long-range synchronous fast oscillations in the cortex. Nature 383, 621-624.

Uhlhaas, P. J., Linden, D. E. J., Singer, W., Haenschel, C., Lindner, M., Maurer, K., and Rodriguez, E. (2006). Dysfunctional long-range coordination of neural activity during Gestalt perception in schizophrenia. J. Neurosci. 26, 8168-8175.

Uhlhaas, P. J., and Silverstein, S. M. (2005). Perceptual organization in schizophrenia spectrum disorders: a review of empirical research and associated theories. Psychol. Bull. 131, 618-632.

Uhlhaas, P. J., and Singer, W. (2006). Neural synchrony in brain disorders: relevance for cognitive dysfunctions and pathophysiology. Neuron 52, 155-168.

Uhlhaas, P. J., and Singer, W. (2007). What can neural synchrony tell us about autism? Biol. Psychiatry 62, 190-191.

Ullner, E., Vicente, R., Pipa, G., and Garcia-Ojalvo, J. (2009). Contour integration and synchronization in neuronal networks of the visual cortex. Springer Lect. Notes Comput. Sci. Artif. Neural Netw. 5164, 703-712.

Van Vreeswijk, C., Abbott, L. F., and Ermentrout, G. B. (1994). When inhibition not excitation synchronizes neural firing. J. Comput. Neurosci. 1, 313-321.

Varela, F., Lachaux, J.P., Rodriguez, E., and Martinerie, J. (2001). The brainweb: phase synchronization and largescale integration. Nat. Rev. Neurosci. 2, 229-239.

Varela, F. J. (1999). The specious present: a neurophenomenology of time consciousness. In Naturalizing Phenomenology, J. Petitot, F. J. Varela, J.-M. Roy, and B. Pachoud, eds.
(Stanford, CA, Stanford University Press), pp. 266-314.

Vicente, R., Gollo, L. L., Mirasso, C. R., Fischer, I., and Pipa, G. (2008). Dynamical relaying can yield zero time lag neuronal synchrony despite long conduction delays. Proc. Natl. Acad. Sci. U.S.A. 105, 17157-17162.

Villalobos, M. E., Mizuno, A., Dahl, B. C., Kemmotsu, N., and Muller, R. A. (2005). Reduced functional connectivity between $\mathrm{V} 1$ and inferior frontal cortex associated with visuomotor performance in autism. NeuroImage $25,916-925$.

Volgushev, M., Chistiakova, M., and Singer, W. (1998). Modification of discharge patterns of neocortical neurons byinduced oscillations of the membrane potential. Neuroscience 83, 15-25.

von Stein, A., Chiang, C., and König, P. (2000). Top-down processing mediated by interareal synchronization. Proc. Natl. Acad. Sci. U.S.A. 97, 14748-14753.

Williams, S. R., and Atkinson, S. (2008). Dendritic synaptic integration in central neurons. Curr. Biol. 22, R1045-R1047.

Wilson, T. W., Rojas, D. C., Reite, M. L., Teale, P. D., and Rogers, S. J. (2007). Children and adolescents with autism exhibit reduced MEG steady-state gamma responses. Biol. Psychiatry 62, 192-197.

Womelsdorf, T., and Fries, P. (2006). Neuronal coherence during selective attentional processing and sensorymotor integration. J. Physiol. (Paris) 100, 182-193.

Womelsdorf, T., Fries, P., Mitra, P., and Desimone, R. (2006). Gamma-band synchronization in visual cortex predicts speed of change detection. Nature 439, 733-736.

Womelsdorf, T., Schoffelen, J.-M., Oostenveld,R.,Singer,W.,Desimone, R., Engel, A. K., and Fries, P. (2007). Modulation of neuronal interactions through neuronal synchronization. Science 316, 1609-1612.

Wu, W., Wheeler, D. W., Staedtler, E. S., Munk, M. H., and Pipa, G. (2008) Behavioral performance modulates spike field coherence in monkey prefrontal cortex. Neuroreport 19, 235-238.

Wynn, J. K., Light, G. A., Breitmeyer, B., Nuechterlein, K. H., and Green, M. F. (2005). Event-related gamma activity in schizophrenia patients during a visual backward-masking task. Am. J. Psychiatry 162, 2330-2336.

Yu,S., Huang, D., Singer,W., and Nikolic, D. (2008). A small world of neuronal synchrony. Cereb. Cortex 18, 2891-2901.

Yuval-Greenberg,S., Tomer,O., Keren, A. S., Nelken, I., and Deouell, L. Y. (2008). Transient induced gamma-band response in EEG as a manifestation of miniature saccades. Neuron 58 , 429-441.

Conflict of Interest Statement: The authors declare that the research was conducted in the absence of any commercial or financial relationships that could be construed as a potential conflict of interest.

Received: 04 March 2009; paper pending published:20March 2009; accepted: 11 July 2009; published online: 30 July 2009. Citation: Uhlhaas PJ, Pipa G, Lima B, Melloni L, Neuenschwander S, Nikolić D and Singer W (2009) Neural synchrony in cortical networks: history, concept and current status. Front. Integr. Neurosci. 3:17. doi: 10.3389/neuro.07.017.2009

Copyright (๑ 2009 Uhlhaas, Pipa, Lima, Melloni, Neuenschwander, Nikolić and Singer. This is an open-access article subject to an exclusive license agreement between the authors and the Frontiers Research Foundation, which permits unrestricted use, distribution, and reproduction in any medium, provided the original authors and source are credited. 